\title{
Multiparticle production and quantum chromodynamics
}

\author{
I.M. Dremin \\ Lebedev Physical Institute, Moscow 119991, Russia
}

\begin{abstract}
The theory of strong interactions, quantum chromodynamics (QCD), is quite successful in the prediction and description of main features of multiparticle production processes at high energies. The general perturbative QCD approach to these processes (mainly to $e^{+} e^{-}$-annihilation) is briefly formulated and its problems are discussed. It is shown that the analytical calculations at the parton level with the low-momentum cut-off reproduce experimental data on the hadronic final state in multiparticle production processes at high energies surprisingly accurately even though the perturbative expansion parameter is not very small. Moreover, it is important that the perturbative QCD has been able not only to describe the existing data but also to predict many bright qualitatively new phenomena.
\end{abstract}

\section{CONTENTS}

1. Introduction

2. QCD equations

3. Comparison with experiment

3.1. The energy dependence of mean multiplicity

3.2. Difference between quark and gluon jets

3.3. Oscillations of cumulant moments

3.4. The hump-backed plateau

3.5. Difference between heavy- and light-quark jets

3.6. Colour coherence in 3 -jet events

3.7. Intermittency and fractality

3.8. The energy behaviour of higher moments of multiplicity distributions

3.9. Subjet multiplicities

3.10. Jet universality

4. Conclusions and outlook

\section{Introduction}

Multiparticle production is the main process of very high energy particle interactions. Studying it, one hopes to get knowledge on the validity of our general ideas about the structure of the matter at smallest distances, on new states of matter which could be created at these extreme conditions, on the asymptotic properties of strong interactions, on the confinement of quarks inside hadrons etc. One should also understand these processes to separate the signals for new physics from the conventional background, in particular, from the features explainable in the framework of quantum chromodynamics. This is necessary for the successful planning of new experiments as well.

With studies of cosmic rays and the subsequent steady increase of energies of particle accelerators, the interest to multiparticle production processes increased as well. The theoretical interpretation of these processes passed through several stages, in detail described in many monographs and review articles. It evolved from the statistical and hydrodynamical approaches to the peripheral, multiperipheral, Regge, parton models and QCD. Let us stress that all of them are somehow used now (quite often in various combinations for the description of different stages of the process) in the computerized Monte Carlo 
The ideas of quantum chromodynamics, where quarks and gluons play a role of partons, nowadays prevail even in the phenomenological models. Neither these phenomenological models nor the widely used analytical perturbative QCD approach can evade an important problem of the transition from partons (quarks, gluons) to the observed particles. This stage is treated phenomenologically within some hypotheses in any of them. It introduces additional parameters which, in principle, can give us a hint to the confinement property, but they sometimes are hard to control and directly extract.

The whole process of multiparticle production is considered as a jet-like cascade of consecutive emissions of partons each of which produces the hadrons observed. Jets from primary quarks were discovered in $e^{+} e^{-}$-collisions in 1975 with the angular distribution expected for spin 1/2 quarks. Gluons emitted by quarks at large transverse momenta can be described by perturbative QCD due to the asymptotic freedom property according to which the coupling strength in QCD decreases with the transferred momentum increasing. Such processes are used to determine the value of the coupling strength. However, one can try to proceed to lower transverse momenta when many jets (and consequently many hadrons) are created. These processes are of main concern for this survey.

Main attention will be paid to the process of $e^{+} e^{-}$-annihilation at high energies, where two pointlike particles collide and the initially created state is determined by the time-like quark-antiquark pair whose evolution gives rise to the production of additional jets. Perturbative QCD has been widely used for the treatment of these processes (as well as for hard jets created in the final states of $e p, p \bar{p}$ and similar interactions). Its predictions and comparison with experimental data are described in the monographs [1, 2, 3, 4, 5] and recent review papers [6, 7, 8, 9, 10]. The application of the QCD ideas to comparatively soft processes in hadronic and nuclear collisions requires for some modification of the approach with account for the internal structure of colliding objects (structure functions) as this is described, e.g., in the surveys [11, 12, 13, 14, 15, 16].

To be compared with experimental data, the results of quantum chromodynamics should be presented either by analytical formulas or numerically as computer calculations according to the Monte Carlo models.

Usually, the Monte Carlo models deal with matrix elements (actually, with the probabilities) of a process at the parton level plus the hadronization stage which uses either the string model [17] or the fragmentation of hadronic clusters [18, 19]. They properly account for the energy-momentum conservation laws because all characteristics of the exclusive process are calculated by computing. One gets all possible characteristics at a given energy but fails to learn their asymptotic behaviour. Let us note that many Monte Carlo models have been proposed which differ by the relative role of the parton and hadronization stages and, correspondingly, by the set of adjusted parameters, which can be interrelated and sometimes are hard to control. Some freedom in their choice also defines the difference in their predictions.

On the contrary, the analytical approach in QCD pretends to start with asymptotic values, where the energy-momentum conservation laws can be neglected in the first approximation, and proceeds to lower energies accounting for conservation laws, higher order perturbative and simplified non-perturbative effects. In the analytical calculations, the perturbative evolution of the jet with the virtuality of the initial parton degrading due to emission of secondary partons is terminated at some low scale $Q_{0}$ of the order of some hundreds $\mathrm{MeV}$ for transverse momenta or virtualities of partons. Some observed variables, e.g., such as the thrust or energy flows, are insensitive to this "infrared" cut-off, i.e. they do not change if collinear or soft partons are additionally emitted.

For others, like the inclusive distributions, the local parton-hadron duality (LPHD) is assumed [20] which declares that the calculated distributions at the parton level describe the hadron observables up to some constant factor. This concept originates [21, 22, 23] from the preconfinement property, i.e. from the local compensation of colour charges, and, consequently, from the tendency of quarks and gluons to form the colourless clusters. Surely, the similarity of the distributions of partons and hadrons substantially owes to the fact that physics of the colour confinement is governed by rather soft processes with small momentum transfers. This works surprisingly well when applied for comparison 
of the theoretical parton distributions with the hadron characteristics observed in experiment. No phenomenological hadronization model is used in this case. At the same time, some specific effects, e.g., the Bose-Einstein correlations for the identical mesons, are out of the scope of this treatment. This is only admissible if these effects are reasonably small.

Even more amazing look two other features of the perturbative QCD approach: the probabilistic description and its applicability to the comparatively soft processes with low transverse momenta円. In the physical gauge, the leading (at high energy) terms appear as the squared moduli of the amplitudes, i.e. as the probabilities. At high energies, the quantum interference of different amplitudes for the parton production results in the angular (or, more precisely, transverse momentum) ordering of successive emissions of gluons which, in its turn, does not spoil the probabilistic equations for these processes.

The solutions of these equations obtained via the modified perturbative expansion sometimes seem to be applicable even for rather soft processes where the perturbative expansion parameter is not sufficiently small and, moreover, it is multiplied by some large factors increasing with energy. Thus, such an approach can be justified only because some subseries of the purely perturbative expansion ordered according to their high energy behaviour are summed first (from here originates the term "modified perturbation theory") and then the asymptotic series is cut off at the proper order.

Surely, the probabilistic treatment is just the simplest approach to the whole description of the process valid where the colour coherence is not important, the non-linearity of the process, induced, e.g., by the high density of gluons, and, consequently, the unitarization are not taken into account etc. If one has to take these effects into account, then the direct consideration of the traditional Feynman diagrams is preferred because the influence of these specific effects in terms of the generating functional technique is hard to treat. Unfortunately, in this case it becomes more difficult to proceed to the higher order approximations as well. In this framework of the combined study of the equations for the generating functionals and of the interference effects implied by Feynman graphs the perturbative QCD has demonstrated its very high predictive power.

In this paper, we briefly describe the main obtained physical results about the processes of the production of hadronic jets, mostly in $e^{+} e^{-}$-annihilation at high energies, without the detailed discussion of any specific particular technicalities both of the theory and of the experiment. The main goal is to provide the reader with a short guide in this vast region without omission, if this is possible, of main important results and no hiding of newly appearing problems. I am deeply sorry if something essential has been omitted and ask for an excuse from those authors whose papers did not fit the limited space of this review paper.

For example, what concerns the theoretical approaches, after their brief description we demonstrate only the main equations and their solutions with more detailed discussion of the obtained results, their correspondence to experimental data and physical meaning. This would allow the reader to get quickly acquainted with the general situation and then, following the numerous cited original papers, more voluminous surveys and monographs, to study it in more detail if some interest to the particular problem has appeared. The comparison of theoretical results with experimental data is demonstrated in some figures which constitute only a small part of those available in the papers referred to.

Thus this review paper is mainly aimed at those who want to learn about the achievements and problems in the applications of quantum chromodynamics to the description of multiparticle production processes at high energies, especially, those who start their study of high energy particle physics or specialists in other fields.

\section{QCD equations}

The most general calculational approach to the characteristics of multiparticle production processes starts from the expression for the generating functional [24, 25], the equations governing its evolution

\footnotetext{
${ }^{1}$ For more details see Refs. [5, 9]
} 
in the framework of the considered theoretical scheme and the subsequent solution of these equations. The generating functional contains the complete information about any multiparticle process and is defined as

$$
G(u, y)=\sum_{n} \int d^{3} k_{1} \ldots d^{3} k_{n} u\left(k_{1}\right) \ldots u\left(k_{n}\right) P_{n}\left(k_{1}, \ldots, k_{n} ; y\right),
$$

where $P_{n}\left(k_{1}, \ldots, k_{n} ; y\right)$ is the probability density for the exclusive production of $n$ particles with momenta $k_{1}, \ldots, k_{n}$ at the initial virtuality (energy), proportional to $\exp (y)$, and $u(k)$ is an auxiliary function. For $u(k)=$ const, one gets the generating function of the multiplicity distribution $P_{n}(y)$ :

$$
G(u, y)=\sum_{n=0}^{n=\infty} u^{n} P_{n}(y) .
$$

The variational derivatives of $G(\{u\})$ over $u(k)$ (or usual $u$-derivatives for constant $u$ ) provide any inclusive and exclusive distributions (in particular, the average values and correlators of any rank), i.e. the complete information about all characteristics of the process. This information is, however, unavailable unless the equations for the evolution of the generating functional are formulated. These equations depend, naturally, on the theory which has been used for the description of these processes.

Two equations have been proposed in quantum chromodynamics to describe the multiparticle production processes in the different kinematical regions. In brief they are named by the first letters of the names of their authors. They are the DGLAP equation [26, 27, 28] and the BFKL equation [29, 30, 31]. The DGLAP equation applies to the process of the evolution of the virtualities (or transverse momenta) of the system. The BFKL equation deals with this evolution in terms of the longitudinal momenta. Correspondingly, the DGLAP equations are used for the description of the development of the partonic jets where the initial highly virtual time-like parton] evolves, e.g., as in $e^{+} e^{-}$-annihilation at high energies or hard jets in hadronic processes (see the review papers [6, [, 8, 9, 10]). In its turn, the longitudinal

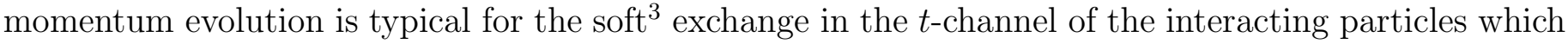
is of the multiperipheral character with some rescatterings (see the review papers [11, 12, 13, 14, 15, 16]). Some proposals for the interpolation between these two regions have been promoted [32, 33].

Initially, these equations were derived in the framework of the Feynman diagram technique by the summation of certain subseries from the whole perturbation series expansion. Their validity has been proven in the leading and next-to-leading approximations of the modified perturbation theory (in more detail it will be described later). Both equations are applicable to the processes in which the density of the gluon field is comparatively low and the multiparton interactions and screening may be neglected. They have been successfully applied to interpretation of many experimental results and for prediction of new properties of various distributions of secondary particles.

However, these simplified versions of both equations have many unsolved problems. They are partly described below in the case of the DGLAP equation. What concerns the BFKL equation, let us just mention only one, however important, problem related to the prediction of the power-like increase of the total cross sections with energy which violates the unitarity condition and contradicts to the Froissart bound. According to this bound, the total cross sections of hadronic processes are allowed to increase with energy $s$ not faster than $\sigma \propto \log ^{2} s$. In the leading order of the modified perturbation theory, the BFKL equation predicts the power-like increase of the total cross sections with very high exponent $\sigma \propto s^{0.5}$, that strongly contradicts not only to theoretical restrictions but also to all modern experimental results. Recently, it was shown [34, 35] that the corrections due to the next order terms are quite large, and even though the power dependence of cross sections on energy persists, the exponent becomes much lower $\sigma \propto s^{0.17}$. Such an increase is already much closer to the phenomenological fits of experimental data at presently available energies which give rise to the exponent values in the range from 0.08 to 0.12 . One can hope that the higher order corrections will lead to further decrease of the

\footnotetext{
${ }^{2}$ With large positive 4-momentum squared $Q^{2}>0$.

${ }^{3}$ When the longitudinal momentum transfers $x$ are shared by small portions; $x \ll 1$.
} 
exponent while the summation of the subseries of the perturbative expansion would be able to avoid the contradiction with the Froissart condition.

The unified field-theoretical description of both approaches can be obtained if the proper effective action of the theory [36, 37, 38, 39, 34, 35] is constructed. In particular, in the papers [36, 37, 38, 39] apart from the term of the standard Yang-Mills gluonic action, the effective action contains the term corresponding to the non-abelian eikonal interaction of the fastly moving sources with the relevant component of the gluon field. Such field-theoretical scheme with the effective action admits the generalization of these equations to the non-linear case. In the framework of the Wilson renormalization group approach one can derive the unified functional QCD equation [37, 38] in the leading logarithmic approximation which takes into account the higher orders of the parton density fluctuations. It leads to the system of interconnected equations for the partonic correlators of an arbitrary order. However, a lot of work is still needed in this direction as to develop the higher order approximation approach as to understand the role of the mechanism of the non-linear contributions and the unitarity bounds.

As previously mentioned, this review is mainly devoted to the $e^{+} e^{-}$-annihilation processes where the experimental data are most precise and extensive ones. Therefore the solution of the DGLAP equations for the evolution of partonic jets constitutes the main content of the theoretical approach adopted heref.

The general structure of the equation for the generating functional in QCD describing the jet evolution for single species partons can be written symbolically as

$$
G^{\prime} \sim \int \alpha_{S} K[G \otimes G-G] d \Omega
$$

It shows that the evolution of the functional $G$ indicated by its derivative $G^{\prime}$ over the evolution parameter (the transverse momentum or the virtuality) is determined by the cascade process of the production of two partons by a highly virtual time-like parton (the term $G \otimes G$ ) which provides new partons in the phase space volume considered $d \Omega$ and by the escape of a single parton $(G)$ from a given phase space region.

Therefore this equation contains terms corresponding to inflow and outflow of partons. In fact, it can be interpreted as the kinetic equation with the collision integral in the right hand side. The weight factors are determined by the coupling strength $\alpha_{S}$ and the splitting function $K$ which is defined by the interaction Lagrangian. The integral runs over all internal variables, and the symbol $\otimes$ shows that the two created partons share the momentum of their parent. The initial condition for equation (3) is defined by the requirement for the jet to be created by a single initial parton, i.e., by

$$
P_{n}=\delta_{n 1} ; \quad G_{0}=u(k)
$$

It is clear from this formula that we have to deal with the non-linear integro-differential probabilistic equation with shifted arguments in the $G \otimes G$ term under the integral sign.

For quark and gluon jets, one writes down the system of two coupled equations. Their solutions give all characteristics of quark and gluon jets and allow for the comparison with experiment to be done. Let us write now them down explicitly for the generating functions:

$$
\begin{aligned}
G_{G}^{\prime} & =\int_{0}^{1} d x K_{G}^{G}(x) \gamma_{0}^{2}\left[G_{G}(y+\ln x) G_{G}(y+\ln (1-x))-G_{G}(y)\right] \\
+ & n_{f} \int_{0}^{1} d x K_{G}^{F}(x) \gamma_{0}^{2}\left[G_{F}(y+\ln x) G_{F}(y+\ln (1-x))-G_{G}(y)\right], \\
G_{F}^{\prime} & =\int_{0}^{1} d x K_{F}^{G}(x) \gamma_{0}^{2}\left[G_{G}(y+\ln x) G_{F}(y+\ln (1-x))-G_{F}(y)\right],
\end{aligned}
$$

\footnotetext{
${ }^{4}$ In the forthcoming issues of the Physics-Uspekhi journal, it is intended to publish the surveys on the effective action, on description of soft processes in quantum chromodynamics (physics of small $x$ and its relation to the Regge approach).
} 
where $G^{\prime}(y)=d G / d y, y=\ln \left(p \Theta / Q_{0}\right)=\ln \left(2 Q / Q_{0}\right)$ is the evolution parameter, defining the energy scale, $p$ is the initial momentum, $\Theta$ is the angle of the divergence of the jet (jet opening angle), assumed here to be fixed, $Q$ is the jet virtuality, $Q_{0}=$ const, $n_{f}$ is the number of active flavours,

$$
\gamma_{0}^{2}=\frac{2 N_{c} \alpha_{S}}{\pi}
$$

the running coupling constant in the two-loop approximation is

$$
\alpha_{S}(y)=\frac{2 \pi}{\beta_{0} y}\left(1-\frac{\beta_{1}}{\beta_{0}^{2}} \cdot \frac{\ln 2 y}{y}\right)+O\left(y^{-3}\right),
$$

where

$$
\beta_{0}=\frac{11 N_{c}-2 n_{f}}{3}, \quad \beta_{1}=\frac{17 N_{c}^{2}-n_{f}\left(5 N_{c}+3 C_{F}\right)}{3},
$$

the labels $G$ and $F$ correspond to gluons and quarks, and the kernels of the equations are

$$
\begin{gathered}
K_{G}^{G}(x)=\frac{1}{x}-(1-x)[2-x(1-x)], \\
K_{G}^{F}(x)=\frac{1}{4 N_{c}}\left[x^{2}+(1-x)^{2}\right], \\
K_{F}^{G}(x)=\frac{C_{F}}{N_{c}}\left[\frac{1}{x}-1+\frac{x}{2}\right],
\end{gathered}
$$

$N_{c}=3$ is the number of colours, and $C_{F}=\left(N_{c}^{2}-1\right) / 2 N_{c}=4 / 3$ in QCD. The asymmetric form (10) of the three-gluon vertex can be used due to the symmetry properties of the whole expression. If one puts $n_{f}=0$ in equation (5) and omits equation (6), then one gets the equation of gluodynamics briefly described above. The auxiliary variable $u$ has been omitted in the generating functions for simplicity.

The typical feature of any field theory with a dimensionless coupling constant (quantum chromodynamics, in particular) is the presence of the singular terms at $x \rightarrow 0$ in the kernels (10), (12) of the equations. They imply the uneven sharing of energy between newly created jets and play an important role in the jet evolution giving rise to its more intensive development compared with the equal proportions (nonsingular) case.

Let us note that these equations can be transformed into the linear equations for the moments of multiplicity distributions (see subsections 3.1 and 3.3). For the running coupling strength (8), they have been solved only in the perturbative theory approach. The systematical method, proposed in 40], consists in using the Taylor series expansion of the expressions under the integral sign. It results in the modified perturbative expansion of the physically measurable quantities. Namely such solutions are usually considered when the analytical formulas are compared with experimental results.

At the same time, these equations can be exactly solved [41, 42 if the coupling strength is assumed fixed, independent of $y$, i.e. $\alpha_{S}=$ const instead of (8). This becomes possible because of the scaling property leading to the power-like behaviour of the average multiplicity and all higher moments for the fixed coupling case. This behaviour differs from the dependences obtained in the case of the running coupling strength (see subsection 3.1). Therefore, the slow logarithmical decrease of this strength with transferred momenta increasing is crucial for the development of the parton cascades.

Even though the system of equations (5), (6) is physically appealing, it is not absolutely exact, i.e., it is not derived from the first principles of quantum chromodynamics. One immediately notices this since, for example, in these equations there is no four-gluon interaction term which is contained in the lagrangian of QCD. This interaction should, probably, correspond to the contribution to the integral term of the equations proportional to the product of three generating functions with a corresponding weight factor. Such a term does not contribute the singularity to the kernels and its omission is justified 
in the lowest order approximations. There were no attempts to take this term into account in the higher order perturbative expressions.

Nevertheless, the modified series of the perturbation theory with three-parton vertices is well reproduced by such equations up to the terms including two-loop and three-loop corrections. As shown in Ref. [5], the neglected terms would contribute at the level of the product of, at least, five generating functions. The physical interpretation of the corresponding Feynman graphs would lead to the treatment of the 'colour polarizability' of jets.

These equations are justified up to some approximation of the modified perturbation theory (see below), because they only include those Feynman diagrams, where the gluons have strongly ordered transverse momenta, and are not close to the kinematical limit. Apart from this, they take into account the non-perturbative effects (e.g., the properties of the QCD vacuum) only in a simplified manner by the direct cut-off of the cascade evolution at some virtuality $Q_{0}$. In principle, the effective infrared-safe coupling constant (without the Landau pole) 43 may be used as the substitute for the phenomenological parameter $Q_{0}$. It must be universal for different processes and tend to a constant limit at low virtualities. The constant average value of the coupling strength in this region has been used in papers [44, 45] dealing with the non-perturbative corrections. However, the behaviour of the coupling strength is not the only non-perturbative effect. Therefore, we will use the more traditional perturbative approach. The non-perturbative effects in the three-jet events have been studied in more detail in the papers 46.

There are some problems also with the definition of the evolution parameter, with preasymptotic corrections etc. For example, the lower and upper limits of integration over $x$ in Eqns (5), (6) are constant and correspond to their asymptotic values. In reality, they vary in the preasymptotic region. Their form is determined by the restriction imposed on the transverse momentum which is given by the inequality

$$
k_{t}=x(1-x) p \Theta^{\prime}>Q_{0} / 2 .
$$

This condition originates from the requirement that the formation time of a gluon $\left(t_{\text {form }} \sim k / k_{t}^{2}\right)$ must be less than its hadronisation time $\left(t_{\text {had }} \sim k R^{2} \sim k / Q_{0}^{2}\right)$. It should be imposed for the perturbative QCD to be applicable. This leads to the requirement that the arguments of the generating functions in Eqns (5), (6) should be positive. Therefore, we must integrate in Eqns (5), (6) over $x$ from $\exp (-y)$ to $1-\exp (-y)$. However these limits tend to 0 and 1 at high energies $(y \rightarrow \infty)$. The omitted contributions decrease in the power-like manner with energy increasing. That is why it seems reasonable to learn more about the solutions of equations (5), (5) near the asymptotic region taking first into account the perturbative (logarithmically decreasing) corrections, and only then take the neglected power-like terms into account as further corrections to these solutions.

Moreover, this cut-off of the limits of integration is of physical importance. With the limits equal to $\exp (-y)$ and $1-\exp (-y)$, the partonic cascade terminates at the perturbative level $Q_{0} / 2$ as is seen from the arguments of the generating functions in the integrals. With the limits equal to 0 and 1 , one extends the cascade into the non-perturbative region with low virtualities $Q_{1} \approx x p \Theta / 2$ and $Q_{2} \approx(1-x) p \Theta / 2$ less than $Q_{0} / 2$. Namely this region contributes terms of the order of $\exp (-y)$, power-suppressed in energy. It is not clear whether the equations and LPHD hypothesis are valid down to some $Q_{0}$ only or the non-perturbative region can be included as well.

Some approximations are used to solve these equations with the running coupling strength. The Taylor series expansion leads [40] immediately, as will be shown below, to the perturbation theory series in the exponent of the physically measurable quantities. It implies the summation of some specific subseries of the purely perturbative expansion in the coupling strength for this particular characteristics. This justifies the term of the "modified perturbative expansion" attached to this procedure. Moreover, the expansion parameter is not $\alpha_{S}$ itself but its square root $\gamma_{0}$. The asymptotic results (for extremely high energies) are obtained in the so-called double-logarithmic (DLA or DLLA if it is called as doubleleading-logarithmic) or leading order (LO) approximation where the terms $\left(\alpha_{S} \ln ^{2} s\right)^{n}$ are summed. Here $s$ is the cms energy squared. The emitted gluons are assumed to be so soft that the energy-momentum 
The corrections accounting for conservation laws in the $G \otimes G$ term, i.e., the shift in their arguments in equations (5), (6), as well as the higher order terms in the weight $\alpha_{S} K$ (in particular, the non-singular terms of the kernels $K$ and the dependence of $\alpha_{S}$ on the transverse momentum $\left.k_{t}(\sqrt{13})\right)$ appear in the next-to-leading (NLO or MLLA - modified leading logarithmic, NLLA - next-to-leading logarithmic approximation) and higher $(2 \mathrm{NLO}, 3 \mathrm{NLO}, \ldots)$ orders. Formally, these equations have been proven only for the next-to-leading (NLO) order of the modified perturbative QCD. However, one can try to consider them as the kinetic equations and solve them in higher orders with a hope to get from the obtained solutions and their comparison with experiment any indications on the role of the adopted assumptions.

To have a guide to the uncertainties at higher orders of perturbation theory, one can try to generalize these equations by including the abovementioned effects in a more rigorous way than it is usually implied. However, there is no unique way of doing it. Also, it is not clear how one may modify them to include the non-perturbative effects, the colour coherence, the non-linearity at high densities etc, even though the very preliminary phenomenological versions have been proposed [47, 48, 32, 49, 50, 51, 52, 33, 53. The simplest proposal would be to compare two alternative evolution equations which use somewhat different assumptions leading to different higher order contributions as has been demonstrated in [52, 53].

At the end of the section, let us mention that no direct solution of equations (5), (6) for the generating functions depending on both variables $u$ and $y$ has been obtained. Such solutions would be of interest for finding the location and the strength of the singularities of $G$ in the complex plane $u$. This is important in connection with the behaviour of the moments of the distribution (see formulas (26), (27) below) and with some analogies from the statistical physics, where these singularities would indicate the phase transition point [54, 55]. The corresponding singularities have been found only in the lowest order of the modified perturbation theory. It occured that they are located at the point $u=1+z_{0}$, where $z_{0}=C /\langle n\rangle(C \approx 2.552)$ tends to 0 at high energies. The singular part of $G$ at this location looks like

$$
G(u, y)=\frac{2 z_{0}^{2}}{\left(u-1-z_{0}\right)^{2}}+\frac{2 z_{0}}{u-1-z_{0}}-\frac{2}{3} \log \frac{z_{0}+1-u}{z_{0}}+O(1) .
$$

The structure of singularities is rather complicated. They are close to the point $u=1$ where all moments are calculated. Moreover, with the energy increase they move closer to this point and coincide with it in asymptotics. There is, however, no special reason to worry about it since the generating function should be equal to 1 at this point according to its definition, and, therefore, all singularities must cancel somehow there. This is seen from the above expression as well. Nonetheless, this indicates the completely different structure of the singularities which one obtains in the lowest order perturbative expressions compared with the final result.

In experiment, one always has to cut off the sum over the multiplicity in the definition of the generating function (2) at some maximum multiplicity $n_{\max }$, defined either by the energy-momentum conservation laws or by the definite conditions of a particular experiment. Then the generating function becomes a polynomial of the order of $n_{\max }$ with positive coefficients and, therefore, possesses just this number of the complex conjugated roots. Thus, one can find out the location of the singularity from experimental data only by increasing energy (and, consequently, the value of $n_{\max }$ ) and following the evolution of their locations. According to the papers [54, 55], the complex-conjugated roots of the partition function of the grand canonical ensemble pinch the real axis of $u$ at the limiting transition to the infinite volume just at the singularity location. The behaviour of the same type has been demonstrated in multiparticle production experiments for increasing $n_{\max }$ (in more detail, see Ref. [8]).

\section{Comparison with experiment}

Let us turn now directly to the comparison of the theoretical results obtained with available experimental data. The main bulk of the data is provided by $e^{+} e^{-}$-processes at the $Z^{0}$ energy. Many results can be 
found in the compilation [56].

In experiment, one registers the particles created at the final stage, mostly, hadrons. Then the crude data are corrected for the effectiveness of the detectors, possible radiation of photons before the collision etc. At the same time, as has been mentioned before, the theory provides us with knowledge of the properties of quark and gluon jets and of the distributions of partons created during their evolution. Therefore, the problem of the correspondence of the theoretical results to the experimental data arises. One must separate the jets in an adequate manner and compile the "dictionary" for the translation from the parton to hadron "language".

In $e^{+} e^{-}$-annihilation processes, the quark-antiquark pair is first created. Therefore, the measurement of the multiplicity of the quark jet is of no problem because it is just twice smaller than the total multiplicity and equals to the multiplicity in one of the hemispheres. In terms of the generating functions this can be expressed by the relation

$$
G_{e^{+} e^{-}} \approx G_{F}^{2} .
$$

To get the analogous results about the gluon jets, one should, however, have the access to the pairs of the gluon jets created by a colour-neutral source. This is necessary for the complete correspondence to the theoretical definitions. Unfortunately, such a separation of these events is possible with satisfactorily high precision only either in the decay processes $\Upsilon \rightarrow \gamma g g \rightarrow \gamma$ +hadrons [57] or in the rare collisions leading to the almost parallel heavy quark and antiquark in the same hemisphere with the gluon in the opposite hemisphere [58, 59, 60]. The high experimental statistics at $\mathrm{Z}^{0}$ energy allows to do this. At other energies, where the number of registered events is much smaller, the separation of gluon jets with the help of methods, which do not depend on the chosen algorithm (the so-called "unbiased jets"), became possible quite recently after application of the special analysis of the two- and three-jet events according to two variables - the transverse momentum and energy - proposed in [58, 61, 62] and used in [63] (see the subsection 3.6). The separation of the quark and gluon jets is often done with the help of some special algorithms p. Their use is sometimes not completely identical to the theoretical requirements (the so-called "biased jets"). Then one has to rely only on the comparison with the results of those Monte Carlo models where the applied algorithm has been taken into account. The choice of the algorithm is by itself determined by its physical reliability, by the convenience for the analytical estimates and by the role of the hadronization corrections in these estimates.

What concerns the hadronization stage, i.e., the transition from partons to hadrons, the Monte Carlo models use various phenomenological approaches mentioned briefly above. In the analytical calculations, the variation of the parameter $Q_{0}$ in the combination with the hypothesis of the local parton-hadron duality usually plays the role of the "dictionary" and leads to reasonable results.

\subsection{The energy dependence of mean multiplicity}

The equations for the average multiplicities in jets are obtained from the system of equations (5), (6) by expanding the generating functions in the power series of $u-1$ and keeping only the terms with $q=0$ and 1 with account of the definition of the average multiplicity $\langle n\rangle$ as

$$
\left.\frac{d G}{d u}\right|_{u=1}=\sum n P_{n}=\langle n\rangle .
$$

Finally, one gets the linear integro-differential equations with the shifted arguments under the integral sign for the average multiplicities. They read

$$
\begin{aligned}
\left\langle n_{G}(y)\right\rangle^{\prime}=\int & d x \gamma_{0}^{2}\left[K _ { G } ^ { G } ( x ) \left(\left\langle n_{G}(y+\ln x)\right\rangle+\left\langle n_{G}(y+\ln (1-x)\rangle-\left\langle n_{G}(y)\right\rangle\right)\right.\right. \\
& +n_{f} K_{G}^{F}(x)\left(\left\langle n_{F}(y+\ln x)\right\rangle+\left\langle n_{F}(y+\ln (1-x)\rangle-\left\langle n_{G}(y)\right\rangle\right)\right],
\end{aligned}
$$

\footnotetext{
${ }^{5}$ The most popular one is the method of the jet separation according to their relative transverse momentum called $k_{t^{-}}$
} 


$$
\left\langle n_{F}(y)\right\rangle^{\prime}=\int d x \gamma_{0}^{2} K_{F}^{G}(x)\left(\left\langle n_{G}(y+\ln x)\right\rangle+\left\langle n_{F}(y+\ln (1-x)\rangle-\left\langle n_{F}(y)\right\rangle\right) .\right.
$$

From here, by solving these equations, one can learn about the energy evolution of the ratio of average multiplicities between gluon and quark jets $r$ and of the QCD anomalous dimension $\gamma$ (the slope of the logarithm of average multiplicity in the gluon jet) defined as

$$
r=\frac{\left\langle n_{G}\right\rangle}{\left\langle n_{F}\right\rangle}, \quad \gamma=\frac{\left\langle n_{G}\right\rangle^{\prime}}{\left\langle n_{G}\right\rangle}=\left(\ln \left\langle n_{G}\right\rangle\right)^{\prime} .
$$

They have been represented by the perturbative expansion at large energy (or large $y$ ) as

$$
\begin{gathered}
\gamma=\gamma_{0}\left(1-a_{1} \gamma_{0}-a_{2} \gamma_{0}^{2}-a_{3} \gamma_{0}^{3}\right)+O\left(\gamma_{0}^{5}\right), \\
r=r_{0}\left(1-r_{1} \gamma_{0}-r_{2} \gamma_{0}^{2}-r_{3} \gamma_{0}^{3}\right)+O\left(\gamma_{0}^{4}\right) .
\end{gathered}
$$

Using the Taylor series expansion [40] of $\langle n\rangle$ at large $y$ in Eqns (17), (18) with (20), (21) and equating the terms of the same order in $\gamma_{0}$ in both sides, one gets the coefficients $a_{i}, r_{i}$ shown in the Table 1 $\left(r_{0}=9 / 4\right)$.

Table 1
\begin{tabular}{|c|c|c|c|c|c|c|}
\hline$n_{f}$ & $r_{1}$ & $r_{2}$ & $r_{3}$ & $a_{1}$ & $a_{2}$ & $a_{3}$ \\
\hline 3 & 0.185 & 0.426 & 0.189 & 0.280 & -0.379 & 0.209 \\
\hline 4 & 0.191 & 0.468 & 0.080 & 0.297 & -0.339 & 0.162 \\
\hline 5 & 0.198 & 0.510 & -0.041 & 0.314 & -0.301 & 0.112 \\
\hline
\end{tabular}

The parameter $\gamma$ determines the exponent of the mean multiplicity of the gluon jet

$$
\left\langle n_{G}\right\rangle=\exp \left(\int^{y} \gamma\left(y^{\prime}\right) d y^{\prime}\right)
$$

and therefore its perturbative expansion in terms of $\gamma_{0}$ corresponds in each particular order in $\gamma_{0}$ to the summation of some subseries in the purely perturbative expression for the average multiplicity, i.e., to the modified perturbation theory for this particular physical quantity. The choice of the gluon jet is related, first of all, with the tradition to study in the beginning the limiting case of gluodynamics where $n_{f}=0$, i.e., quarks and, correspondingly, equation (18) are not cosidered. This is justified also because in the lowest order approximations (the NLO corrections including) the energy dependence of average multiplicities in quark and gluon jets do not differ (for more detail, see next subsection).

One of the most spectacular predictions of QCD states that in the leading order approximation (i.e., asymptotically), where $\gamma=\gamma_{0}$, average multiplicities should increase with energy [65, 66, 67] like $\exp [2 c \sqrt{\log s}]$ with the theoretically calculable value of $c$. This behaviour is just in between the power-like and logarithmical dependences correspondingly predicted by the hydrodynamical and multiperipheral models]. Next-to-leading order results account for the term with $a_{1}$ in Eqn. (20) [68, 69, 70] and contribute the logarithmically decreasing factor to this behaviour. Namely these two terms determine the main energy dependence of average multiplicities, and they are the same for quark and gluon jets. The higher order terms do not practically change this dependence [71, 72]:

$$
\begin{array}{r}
\left\langle n_{G}\right\rangle=K y^{-a_{1} c^{2}} \exp \left(2 c \sqrt{y}+\frac{c}{\sqrt{y}}\left[2 a_{2} c^{2}+\frac{\beta_{1}}{\beta_{0}^{2}}(\ln 2 y+2)\right]\right. \\
\left.+\frac{c^{2}}{y}\left[a_{3} c^{2}-\frac{a_{1} \beta_{1}}{\beta_{0}^{2}}(\ln 2 y+1)\right]\right),
\end{array}
$$

where $c=\left(4 N_{c} / \beta_{0}\right)^{1 / 2}$. The gluodynamics expressions can be obtained from this formula for $n_{f}=0$ taking into account it also in the analytical expressions for $a_{i}$ given in [72].

${ }^{6}$ It is not excluded, however, that the non-linear corrections which appear, say, due to the high gluonic density can unitarize this approximation and give rise to the logarithmic increase of the mean multiplicity in the asymptopia 
The fitted parameters in the final expression are the overall constant normalization factor $K$ which is defined by the confinement[ and the scale parameter $Q_{0}=2 \Lambda$. The $e^{+} e^{-}$-data in the energy interval from the $\Upsilon$-resonance to LEP-2 (i.e., approximately from 10 to $200 \mathrm{GeV}$ ) are well fitted by such an expression as seen in Fig. 1. The dotted line corresponds to the fit with two adjustable parameters. The dashed line shows the fit by the Monte Carlo model HERWIG. Other lines determined by the difference between gluon and quark jets will be explained in next subsection.

Let us note here that the expansion parameter $\gamma$ is rather large at present energies ranging from 0.4 to 0.5. The obtained expressions are valid at high energies and do not pretend to describe the energy behaviour of mean multiplicities near threshold. The computer solutions [73, 74] of equations (5), (6) give rise to the satisfactorily precise agreement with experimental data even at rather low energies.

Equations (17), (18) can be solved exactly in the case of the fixed coupling constant [41, 42] because of the scaling property according to which the relation

$$
\frac{\langle n(y+\ln x)\rangle}{\langle n(y)\rangle}=x^{\gamma}
$$

is valid for $\gamma_{0}=$ const and integro-differential equations (17), (18) are reduced to the system of easily solvable linear algebraic equations. The energy increase of mean multiplicity becomes power-like because the exponent acquires the general structure of the type of $\gamma_{0} \ln s$.

\subsection{Difference between quark and gluon jets}

The system of two equations for quark and gluon jets predicts that asymptotically the energy dependence of mean multiplicities for them should be identical. Moreover, this coincidence is exact in the next-toleading approximation of the modified perturbation theory as well. Higher order correction, even though violating this beautiful feature, are comparatively weak in the functional dependence on energy. Namely this explains the initial success in the description of the energy dependence of average multiplicities in $e^{+} e^{-}$-annihilation in the framework of gluodynamics.

The absolute normalization is not fixed. However, the relative normalization, as given by $r$ (21), is calculable. The gluon jets are more "active" than the quark jets so that the ratio $r=\left\langle n_{G}\right\rangle /\left\langle n_{F}\right\rangle$ of average multiplicities between gluon and quark jets should tend at high energies [75] to the ratio of the Casimir operators $C_{A} / C_{F}=9 / 4$.

Once again, by comparison with experiment this prediction shows how far are we now from the true asymptotics. Even though this prediction obtained in the leading order (LO) approximation is valid qualitatively, its quantitative value is still rather far from experimental ones where this ratio is about 1.5 at $Z^{0}$ energy and even smaller at lower energies. The higher order ${ }^{8}$ terms [76, 77, 78, 68, 72, 79] are rather important just for this parameter. They have been calculated now up to 3NLO (or 4NLO; see the footnote) terms (see Table 1) and improve the agreement approaching the experimental value with an accuracy about 15\% (see Fig. 2 where the old notation for the approximation order has been used). Let us stress again that just the ratio of mean multiplicities $r$ (but not the energy behaviour of any of them, $\left\langle n_{G}\right\rangle$ or $\left\langle n_{F}\right\rangle$, separately) is most sensitive to these corrections. This is because the main LO and NLO terms cancel there and decline from the constant value $r_{0}$ is governed by the higher order terms. This decline can provide a guide to further generalizations of the equations and to the proper account of the nonperturbative contributions.

Surely, the higher order terms change slightly also the energy behaviour of multiplicities for quark

\footnotetext{
${ }^{7}$ That is why the lower limit of the integration over $y^{\prime}$ in formula (22) is not fixed.

${ }^{8}$ The difference in the definition of the "order" for the anomalous dimension $\gamma$ and for the ratio of mean multiplicities $r$ is described in Ref. [72]. In particular, the term $r_{3}$ in $r$ should be actually considered as 4NLO correction since it is added to the still uncalculated term $a_{4}$ in the higher order expression for the quark jet anomalous dimension $\gamma_{F}$ and, consequently, influences its multiplicity.
} 
jets compared to gluon jets'] as observed in experiment. However, the simultaneous fit of quark and gluon jets with the same set of fitted parameters even in the framework of 3NLO approximation is still not very accurate as is seen from the shaded area in Fig. 1. This area demonstrates the attempt of such a description with the data on gluon jets divided by the theoretically (analytically) calculated value of the ratio $r$. Its failure shown by the shift of the shaded area compared to the experimental points and by its large width is again due to the insufficiently precise description of the ratio $r$. The agreement with experimental multiplicity in $e^{+} e^{-}$is restored either in the case of the normalization according to gluon jets with the only adjusted parameter $Q_{0}=2 \Lambda$ (the solid line in Fig. 1) or in the case with both parameters fitted (the dotted line).

The computer solution of the equations [74 gives rise to very good agreement with experiment at the $Z^{0}$ resonance and leaves a rather small difference about $20 \%$ even at so low energies as the mass of the $\Upsilon$. In the analytical approach, the quite good agreement on the ratio $r$ at the energy of the $Z^{0}$ has been achieved when the equations are modified to account for the phase space limitations imposed by energy-momentum conservation in the dipole cascade picture of the string approach [52]. However, some problems arise for higher moments of the multiplicity distribution in such an approach [53].

Equation (21) states that the ratio $r$ increases with energy increasing due to the decrease of $\gamma_{0}$ (the asymptotic freedom) but its slope becomes smaller. Experimental values also increase with energy as seen from Fig. 2. There are common tendencies and good qualitative agreement between the results of analytical calculations and experiment on the value of the ratio $r$. However, the quantitative statements are valid only within the $15-25 \%$ accuracy.

More detailed characteristics of jets behaviour can be obtained from the slope and the curvature of energy dependences of multiplicities [80, 72]. Some experimental data about it have been recently obtained 63.

The slope of the energy dependence of the ratio of average multiplicities between gluon and quark jets $r$, i.e., the derivative $r^{\prime}$ ("the slope of the ratio"), decreases with the energy increase according to the expansion (21) as $\ln ^{-3 / 2} s$. It is very sensitive to the higher order corrections 80, 72, first of all, due to the large value of the ratio $r_{2} / r_{1}$ :

$$
r^{\prime}=B r_{0} r_{1} \gamma_{0}^{3}\left[1+\frac{2 r_{2} \gamma_{0}}{r_{1}}+\left(\frac{3 r_{3}}{r_{1}}+B_{1}\right) \gamma_{0}^{2}+O\left(\gamma_{0}^{3}\right)\right]
$$

where $B=\beta_{0} / 8 N_{c}, B_{1}=\beta_{1} / 4 N_{c} \beta_{0}$. Therefore its use for comparison with experiment does not seem to be informative enough.

At the same time, such corrections are partly cancelled in "the ratio of the slopes" $r^{(1)}=\left\langle n_{G}\right\rangle^{\prime} /\left\langle n_{F}\right\rangle^{\prime}$ for gluon and quark jets [80, 72]. Thus, the ratio of the slopes must depend on energy much weaker. This has been observed in experiment. The first comparison [8] of the theoretical estimates of this ratio [81, 82] with experiment has shown quite good agreement, however, still within rather large undefiniteness in experimental results. Recent more accurate separation of unbiased gluon jets 63] made it possible to get the values not only the ratios $r$ and $r^{(1)}$ at energies from $\Upsilon$ to $\mathrm{Z}^{0}$ (including the intermediate ones) but also the values of the ratio of the second derivatives $r^{(2)}=\left\langle n_{G}\right\rangle^{\prime \prime} /\left\langle n_{F}\right\rangle^{\prime \prime}$. The asymptotic values for all of them are predicted equal 2.25. The corresponding values at the energy of the hadronic component in $g g$-system $30 \mathrm{GeV}$ are $r=1.422 \pm 0.051, r^{(1)}=1.761 \pm 0.071, r^{(2)}=1.92 \pm 0.13$ and at $80 \mathrm{GeV} r=1.548 \pm 0.042, r^{(1)}=1.834 \pm 0.09, r^{(2)}=2.04 \pm 0.14$. The error bars are mainly determined by the systematic errors. These results are in agreement with the analytical QCD predictions [80, 72] that at present energies $r^{(2)}$ should be closer to its asymptotic value of 2.25 than $r^{(1)}$, and $r^{(1)}$ closer to 2.25 than $r$, i.e. the inequalities $r<r^{(1)}<r^{(2)}$ are valid. The experimentally obtained values of the ratios of slopes and curvatures of energy dependence of gluon and quark jets and their comparison with some theoretical calculations are demonstrated in Figs. 3 and 4.

\footnotetext{
${ }^{9}$ The value $\gamma$ in $(22)$ is replaced there by $\gamma_{F}=\gamma-r^{\prime} / r$, which differs from $\gamma$ only in higher orders as is easily estimated if one recalls that $r^{\prime} \sim \gamma_{0}^{\prime} \sim \gamma_{0}^{3}$ (see below formula (25)).
} 
One can hope that the asymptotic values of the multiplicities ratio $r$ can be approached closer if the soft particles are only considered with energies much lower than the total energy of the jet [83, 84]. This effect has been really noticed but it is not very strong. The ratio $r$ for particles with low momenta is about 1.8 [85].

It is interesting that in the Monte Carlo model HERWIG accurately reproducing this ratio for soft hadrons the corresponding ratio for soft partons occurs exactly equal to 2.25. Thus the role of the hadronization stage is very important for the ratio $r$ in this particular model. Let us note that this situation differs from the case where all momenta are averaged as demonstrated in Fig. 2 with the lines of the HERWIG model practically indistinguishable for partons and particles. This is related to the strong difference of the spectra which are rather soft for hadrons and much harder for partons in this model (for more detail, see Ref. [8]). The direct use of the local parton-hadron duality is, therefore, impossible here because of the large value of the adopted cut-off parameter $Q_{0}$ indicating the early finish of the parton stage

The parton stage in the evolution of the jet of final hadrons can be separated in a following way. In the HERWIG model it is assumed to finish at $Q_{0}=0.8 \mathrm{GeV}$. Thus it corresponds to the rather large transverse momenta. Therefore one should also choose the hadrons with large transverse momenta. Analysis of the data about the comparatively soft hadrons $(p<4 / c)$ with rather large transverse momenta $p_{t}>0.8 / c$ have led to the result 85] for the ratio $r=2.29 \pm 0.017$ in this region which agrees with asymptotic predictions. In its turn, this result could agree with the computer solutions of equations (5), (6) and with the local parton-hadron duality hypothesis only if one adopts that the evolution of the "partonic" cascade in the computer calculations to the region $Q_{0}<0.8 \mathrm{GeV}$ plays the same role as the "hadronization" stage in the HERWIG model, where such an evolution is discarded.

The widths of the multiplicity distributions differ for quark and gluon jets, the former being somewhat wider. Qualitatively, QCD describes this tendency but quantitative estimates are rather uncertain yet as is discussed in more detail in subsection 3.8 .

\subsection{Oscillations of cumulant moments}

The shape of the multiplicity distribution can be described by its higher moments related to the width, the skewness, the kurtosis etc. Equations for the moments of the distributions of the $q$-th rank are obtained from the system of equations (5), (6) by comparing the terms of the type $(u-1)^{q}$ in both sides of equations. The $q$-th derivative of the generating function corresponds to the factorial moment $F_{q}$, and the derivative of its logarithm defines the so-called cumulant moment $K_{q}$. The latter ones describe the genuine correlations in the system irreducible to the products of lower order correlations (this recalls the connected Feynman graphs).

$$
\begin{gathered}
F_{q}=\frac{\sum_{n} P_{n} n(n-1) \ldots(n-q+1)}{\left(\sum_{n} P_{n} n\right)^{q}}=\left.\frac{1}{\langle n\rangle^{q}} \cdot \frac{d^{q} G(z)}{d u^{q}}\right|_{u=1}, \\
K_{q}=\left.\frac{1}{\langle n\rangle^{q}} \cdot \frac{d^{q} \ln G(z)}{d u^{q}}\right|_{u=1} .
\end{gathered}
$$

These moments are not independent. They are connected by definite relations which can easily be derived from their definitions in terms of the generating function:

$$
F_{q}=\sum_{m=0}^{q-1} C_{q-1}^{m} K_{q-m} F_{m} .
$$

They are nothing other than the relations between the derivatives of a function and of its logarithm at the point where the function itself equals 1 . Here

$$
C_{q-1}^{m}=\frac{(q-1) !}{m !(q-m-1) !}=\frac{\Gamma(q)}{\Gamma(m+1) \Gamma(q-m)}=\frac{1}{m B(q, m)}
$$


are the binomial coefficients, and $\Gamma$ and $B$ denote the gamma- and beta-functions, correspondingly. Thus there are only numerical coefficients in recurrence relations (28) and the iterative solution (wellsuited for computer calculations) reproduces all cumulants if the factorial moments are given, and vice versa. In that sense, cumulants and factorial moments are equally suitable for analysis of multiplicity distributions. The relations for the low ranks are

$$
\begin{aligned}
& F_{1}=K_{1}=1, \\
& F_{2}=K_{2}+1, \\
& F_{3}=K_{3}+3 K_{2}+1 .
\end{aligned}
$$

The Taylor series expansion of equations (5), (6) gives rise to the term $G(y)$ in the right-hand side. Dividing by it the left-hand side $G^{\prime}(y)$ one gets the derivative over $y$ of the logarithm of the generating function. The gluodynamics equation in the leading order approximation can be reduced to the differential equation of the second order:

$$
(\ln G(y))^{\prime \prime}=\gamma_{0}^{2}(G(y)-1) ; \quad G(0)=u, \quad G^{\prime}(0)=0 .
$$

After that, the derivatives over $u$ of both sides considered at $u=1$ lead in a natural way to the prediction [40, 41, 79] concerning the behaviour of the ratio $H_{q}=K_{q} / F_{q}$. At asymptotically high energies, this ratio as a function of the rank $q$ is predicted to behave ${ }^{\text {m }}$ as $q^{-2}$. Thus the role of genuine correlations is strongly damped in systems with the large number of particles.

However, the asymptotics is very far from our realm. At present energies, according to QCD, this ratio should reveal the minimum at $q \approx 5$ and subsequent oscillations. In gluodynamics, the minimum is located at

$$
q_{\text {min }} \approx \frac{24}{11 \gamma_{0}}+0.5+O\left(\gamma_{0}\right) .
$$

This astonishing qualitative prediction [40] of the new type of the moments behaviour has been confirmed in experiment (for the very first time in Ref. [86]) as in $e^{+} e^{-}$(see Fig. 5 [87]) as in hadronic processes [88]. The predicted negative minimum of $H_{q}$ is clearly observed. These oscillations can correspond to the replacement of attractive forces (clustering) by repulsion (between clusters) in systems with different number of particles. Let us note that the colour coherence in QCD discussed below also predicts both the attractive and repulsive forces in the systems of the coloured partons.

With energy increasing, one should observe first the disappearence of the oscillations at high ranks and then the smaller dip at the first minimum and its slow shift to higher values of $q$ from its initial position at $q \approx 5$ at $Z^{0}$ energy due to the decrease of $\gamma_{0}$ (see Eqn (32)). Finally, the ratio $H_{q}$ will slowly tend to its asymptotic dependence $q^{-2}$.

The minimum position slowly changes with energy and with the size of the phase space window because it is inverse proportional (see [40, 89]) to the square root of the running coupling strength, i.e., to $\gamma_{0}$. For some specific processes this shift can be very strong (e.g., it has been found for instanton induced processes [90] that the minimum moves to $q \approx 2$ because the multiplicity distribution in these processes is very narrow). At the same time, other experimental data confirm that the oscillations reveal themselves on the qualitative level in various processes, i.e., have some universal origin [88]. The graphical presentation of the ratio $H_{q}$ in place of the moments is suitable, because the moments increase fast with the rank $q$ increasing but this growth cancels in their ratio.

The quantitative analytical estimates are not enough accurate because, first of all, the expansion parameter becomes equal to the product $q \gamma$ which is close to one or even exceeds it for all $q>1$. Therefore the perturbative approach is, strictly speaking, inapplicable to this problem. However, some tricks like Pade-approximation can be used to improve it. At the same time, the numerical computer solution [73, 74] reproduces oscillations quite well.

\footnotetext{
${ }^{11}$ This is clearly seen from equation (31) because each differentiation over $y$ in the left-hand side provides the factor $q \gamma$
} 
These new dependences differ from all those typical for the previously attempted distributions of the probability theory, in particular, such previously popular in particle physics distributions as the Poisson and negative binomial ones. The characteristics which are special for the newly found distribution at high energies have been discussed in Ref. [91].

\subsection{The hump-backed plateau}

Dealing with inclusive distributions of partons within the jet over the rapidity or over the share of the jet energy $(x)$ acquired by the parton, one should solve equations of the type (3) for the generating functional. The variation over the auxiliary function $u(k)$ gives rise to integro-differential equations for the one-particle distributions and the correlation functions. In the leading order in gluodynamics the equation for the distribution function $D(x, y)$ is written as

$$
\frac{d(x D(x, y))}{d y}=\frac{1}{4 N_{c}} \int_{0}^{1} d z \gamma_{0}^{2} K(z)\left[\frac{x}{z} D\left(\frac{x}{z}, y+\ln z\right)\right]
$$

with the boundary condition $x D(x, 0)=\delta(1-x)$. Here, $x=k / E_{j}, k$ is the particle momentum, $E_{j}$ is the jet energy, $K(z)=K_{G}^{G}(z)$. In QCD there are two equations for gluon and quark jets. The subsequent use of the Mellin transformation allows one to reduce these equations in the low orders to the solvable differential equations. This has been done up to the NLO approximation (see [5, 9]).

As predicted by QCD, the momentum (or, more accurately, rapidity $y_{c}^{[2]}$ spectra of the particles inside jets in $e^{+} e^{-}$-annihilation processes should have the shape of the hump-backed plateau [92, 66, [67, 20]. This striking prediction of the perturbative QCD differs from the previously popular flat plateau advocated by Feynman. This has been found in experiment (Fig. 6). The depletion between the two humps is due to the angular ordering and colour coherence in QCD. The humps are of the approximately Gaussian shape (the distorted Gaussian) near their maxima if the variable $\xi=\ln \frac{1}{x}$ is used. This prediction was first obtained in the LO QCD, and more accurate expressions were derived in NLO [93].

The maximum position $\xi_{0}$ in the $\xi$-distribution must shift with energy increase according to the almost logarithmical law with some corrections of the type $O\left(\ln ^{-1 / 2} s\right)$ :

$$
\xi_{0}=y\left[\frac{1}{2}+\sqrt{\frac{C}{y}}-\frac{C}{y}\right]
$$

where $C=\frac{a^{2}}{16 N_{c} \beta_{0}}(\approx 0.3) ; a=\frac{11}{3} N_{c}+\frac{2 n_{f}}{3 N_{c}^{2}}$.

Moments of the distributions up to the fourth rank have been calculated. The spectrum drops quite fast towards smaller momenta. This is a consequence of the colour coherence for the ever softer gluons. It becomes especially noticeable if the variable $\xi$ is used. In the absence of the colour coherence the shape of the distribution near the maxima losts its Gaussian form, the maxima are shifted to the region of smaller values of $x$ (larger $\xi$ ) and, therefore, the spectrum becomes much softer.

The comparison of these QCD predictions with experimental data at different energies has revealed good agreement both on the shape of the spectrum (see Fig. 7 for $e^{+} e^{-}$from 94]) and on the energy dependences of its peak position (see Fig. 8 for $e^{+} e^{-}, e p, p \bar{p}$ from [95]) and of its width.

For soft particles, the spectrum does not depend on the energy [20] and has the shape [96, 97, 83, 84]:

$$
\frac{d n}{d y_{c} d \ln k_{t}} \propto \alpha_{S}\left(k_{t}\right)
$$

This explains the discussed above possibility to observe the approach to the asymptotic value of the ratio $r$ even at finite energies if the soft particles with $p_{t} \rightarrow Q_{0}$ are chosen. The physical origin of this effect lies in the ability of soft partons to resolve only the total charge of the jet (i.e., of the parton initiating it) but not its internal structure. 


\subsection{Difference between heavy- and light-quark jets}

Another spectacular prediction of QCD is the difference between the spectra and multiplicities in jets initiated by heavy and light quarks. Qualitatively, this corresponds to the difference in the bremsstrahlung by muons and electrons where the photon emission at small angles is strongly suppressed for muons because of the large mass in the muon propagator. Therefore, the intensity of the radiation is lower in the proportion to the ratio of the masses squared. The suppression of the photon radiation at small angles was proposed to be exploited [98] also for the top quark where it is especially strong due to its large mass. However, the intensity of the radiation would be too low due to the same reason.

The coherence of soft gluons also plays an important role in QCD. For heavy quarks the accompanying radiation of gluons should be stronger depleted in the forward direction (the so-called dead-cone or ring-like emission). At large angles there should be no visible difference between the emission of gluons by heavy or light quarks. In spite of the close analogy, there is a strong difference between quantum chromodynamics and quantum electrodynamics connected to the fact that the gluons possess the self-interaction while the photons have no point-like interaction of one with another.

It was predicted [99, 100, 101] that the coherence of the soft gluons should result in the energyindependent difference of companion mean multiplicities for heavy- and light-quark jets of equal energy. The companion hadron multiplicity for the heavy quark is defined as the difference between the total multiplicity of the jet initiated by this quark and the constant decay multiplicity $n^{d c}$ in its rest system (which is equal to $11.0 \pm 0.2$ for the $b$-quark and $5.2 \pm 0.3$ for the $c$-quark). Correspondingly, the difference of the total average multiplicities $n$ is written as

$$
n_{Q \bar{Q}}(E)-n_{q \bar{q}}(E)=n_{Q \bar{Q}}^{d c}(M)-n_{q \bar{q}}(\sqrt{e} M)\left[1+O\left(\alpha_{S}(M)\right)\right] .
$$

Here, $q, Q$ denote the light and heavy quarks, $E$ are their energies, $M$ is the heavy quark mass, $\sqrt{e} \approx$ 1.65. The naive model of energy rescaling [102, 103] predicts the decreasing difference at higher energies. The experimental data (see Fig. 9 from [104]) comparing the multiplicities in jets initiated by light $(u, d, s)$ and heavy $(b, c)$ quarks support this QCD conclusion.

Another consequence of such a distinction is the effect of the leading heavy quarks, i.e., the consumption by them of the rather large share of the primary momentum in the process of the emission of gluons. This is related to the suppression of the emission of hard gluons with low transverse momenta which results in the low energy loss by heavy quarks. This effect has also been observed 105 in $e^{+} e^{-}$-annihilation processes. The inclusive spectra of the $b$-quark jet at the energy of the $Z^{0}$ have the maxima at $x_{Q} \approx 0.8-0.9$. In the hadronic processes, this effect was earlier used [106] to explain the so-called "long-flying cascades" in cosmic rays at the energy about $100 \mathrm{TeV}$. In result, the conclusion about the strong increase of the total cross section for the production of heavy quarks in the energy interval up to $100 \mathrm{TeV}$ was obtained. It finds now the direct support from accelerator data.

Also, it has been noticed [107] that the momentum spectra of particles in the $b$-jets are much softer than those in the jets initiated by light quarks as has been predicted in QCD [99] with account of the coherence.

Concerning the difference of the angular distributions for gluon emission by heavy and light quarks initiating the cascade, which lies at the background of all these effects, there exist just the preliminary results [108, 104, which favour the QCD predictions.

Let us note that for hadronic processes the ring-like structure of the polar angle distribution can be observed also for the jets themselves, i.e., for the partons initiating these jets. It can result due to the limitations imposed on the radiation length or due to the so-called Cherenkov gluon effect [109, 110] what corresponds in the "operationalists" language to the emergence of some effective mass.

\subsection{Colour coherence in 3 -jet events}

When three or more partons are involved in a hard interaction, one should take into account colour- 
angles between the two jets [58]. For example, for two jets moving quite close to each other, one should consider their mutual screening. It implies that for the "resolution" of such a pair one should use the rather "hard probe" because the soft ones will feel only the total colour charge of the pair as a whole. In particular, it results in the "colour transparency" of such a pair during its motion inside a hadronic medium. In electrodynamics, the similar effect of the mutual screening of the electric charges of the closely moving electron and positron until they separate at a large distance is known as Chudakov effect [111.

In equation for the generating functionals one should take into account those correlations between the jets which arise if the Feynman diagrams are considered. However, the probabilistic interpretation in the framework of equations (5), (6) in some approximation may fail for these effects. Therefore, for studies of the colour coherence effects the Feynman diagram technique is preferred.

Several such effects have been already observed. In particular, the total multiplicity can not be represented simply as a sum of flows from independent partons. In terms of the observed multiplicities $n_{e^{+} e^{-}}$in annihilation processes, one can write down the multiplicity in the three-jet events $n_{q \bar{q} g}$ [7] as

$$
n_{q \bar{q} g}=\left[n_{e^{+} e^{-}}\left(2 E_{q}\right)+0.5 r\left(p_{t}\right) n_{e^{+} e^{-}}\left(p_{t}\right)\right]\left(1+O\left(\alpha_{S}\right)\right)
$$

where $E_{q}$ is the quark energy and $p_{t}$ is the transverse momentum of the gluon in the center of mass system for the pair $q \bar{q}$. The ratio of the multiplicities for gluon and quark jets is considered at the energy equal to the transverse momentum of the gluon which, in its turn, is related to the virtuality of the quark emitting this gluon. The existence of two scales in the three-jet events is clearly demonstrated in this way. The comparison of formula (37) with experimental data [112, 113 has shown that it is valid for large angles between the jets.

QCD predicts that the particle flows should be enlarged in the directions of emission of partons and suppressed in between them. Especially interesting is the prediction that due to the negative interference this suppression is stronger between the $q \bar{q}$-pair than between $g q$ and $g \bar{q}$ in the hard $e^{+} e^{-} \rightarrow q \bar{q} g$ event if all angles between partons are large. This phenomenon is known as the "string" [114] or "drag" [115] effect. All these predictions have been confirmed by experiment (see Fig. 10 from [116]). In $q \bar{q} g$ events the particle population values in the $q g$ valleys are found larger than in the $q \bar{q}$ valley by a factor $2.23 \pm 0.37$ compared to the theoretical prediction of 2.4. Moreover, QCD predicts that this shape is energy-independent up to an overall normalization factor.

Let us note that for the process $e^{+} e^{-} \rightarrow q \bar{q} \gamma$ the emission of additional photons would be suppressed both in the direction of a primary photon and in the opposite one. In contrast, in the case of the emitted gluon one observes the string (drag) effect of enlarged multiplicity in its direction and stronger suppression in the opposite one. This suppression is described by the ratio of the corresponding multiplicities in the $q \bar{q}$ region

$$
R_{\gamma}=\frac{N_{q \bar{q}}(q \bar{q} g)}{N_{q \bar{q}}(q \bar{q} \gamma)}
$$

which is found to be equal $0.58 \pm 0.06$ in experiment whereas the theoretical prediction is 0.61 .

The colour coherence reveals itself as inside jets as in the inter-jet regions. It should suppress both the total multiplicity of $q \bar{q} g$ events and the particle yield in the transverse to the $q \bar{q} g$ plane for decreasing opening angle between the low-energy jets. When the hard gluon becomes softer, colour coherence determines, e.g., the azimuthal correlations of two gluons in $q \bar{q} g g$ system. In particular, back-to-back configuration $\left(\varphi \sim 180^{\circ}\right)$ is suppressed by a factor $\sim 0.785$ in experiment, 0.8 in HERWIG Monte Carlo and 0.93 in analytical pQCD.

Let us stress once again that the colour coherence determines the topological dependence of the jet properties predefined theoretically in terms of the parton diagrams. The interference between the $q \bar{q}$-pairs not connected by colour is suppressed by the factor $1 / N_{c}^{2}$. This interference is not accounted by the models using the Monte Carlo method. Thus their success in the description of experimental data implies the smallness of such effects. Nevertheless, these small colour-suppressed effects disappearing 
at $N_{c} \rightarrow \infty$ can become really important for the distinction between the analytic diagram approach in QCD and the purely probabilistic Monte Carlo schemes [117. The study of the individual events with very high multiplicity may be crucial for getting the decisive conclusions. The most important lesson derived from the correspondence between the theory and experiment in this case consists in the conclusion that the colour coherence leads to the effects observed at the hadron level and is not wiped out by the hadronization stage. The hadron distributions depend on the topology of the parton stage with hard colour objects that gives further support to the local parton-hadron duality hypothesis.

Some proposals have been promoted for the modification of formula (37) with more correct account for the phase space and for special two-scale (the energy and the transverse momentum) analysis of 3 -jet events when the restriction on the transverse momentum of a gluon jet is imposed [58, 61, 62]. This corresponds to the simultaneous account for the energy and the virtuality of the initially produced quark and antiquark. The first approbation of this proposal [118 has shown the correctness of such a modification. This is related to the solution of the problem of the unique separation of the three-jet events according to the kinematics at the parton level and clear detection of the gluon jet in this system mentioned above. In general, jets are biased because event selection according to some algorithms introduces bias on multiplicities. Jet properties depend on two scales, the transverse momentum and available rapidity range. To compare with theory, one should get unbiased results. For gluon jets, they have been obtained in [63] using the following formula

$$
n_{g g}\left(p_{t}\right)=2\left[n_{q \bar{q} g}\left(s, p_{t}\right)-n_{q \bar{q}}\left(s, p_{t}\right)\right]
$$

where the gluon transverse momentum is given by

$$
p_{t}^{2} s=s_{q g} s_{\bar{q} g}
$$

and $s_{i g}$ is the squared cms energy of the system $i g$. This multiplicity depends on a single variable, whereas the terms in the right hand side depend also on the energies. This implies that there is no dependence on the algorithm of jets separation. It is this accounting of the dependence on the cutoff of the transverse momentum, below which the gluon jet is not resolved and the whole system is treated as a $q \bar{q}$-pair, that finally leads to the independence of the multiplicity of the gluon jet on the adopted algorithm of jet separation.

Let us note that the fast divergence of the modified perturbation series with increase of the term order $n$, which is like $n$ !, usually related to the notion of renormalons [119], gives rise to large nonperturbative corrections. This divergence was noticed above as the appearance of $q \gamma$ factor as the expansion parameter for high ranks of the moments of the distributions.

The non-perturbative corrections to event shapes (especially, to three-jet events) should be mentioned here. Just in this case these corrections are very strong, and there are numerous experimental data here. In distinction to the logarithmic dependences of the perturbative approach, the non-perturbative terms decrease in a power-like manner with increase of the transferred momentum. They can be written in a universal way [120 for various characteristics $\nu_{i}$ as

$$
\delta \nu_{i} \propto c_{i}\left(\Lambda_{e f f}(p) / Q^{2}\right)^{p}
$$

where the parameter $\Lambda_{\text {eff }}$ does not depend on a particular characteristics of the event shape $\nu_{i}$, which can be the jet mass, thrust, jet widening etc. The numerical value of the parameter $c_{i}$ is calculated in QCD [120].

Even though such an approach is quite successful in more precise description of experimental data, there are many unsolved problems. In particular, it is not clear where one should cut off the perturbative series and add to it the non-perturbative terms, what is the relative role of corrections with increasing values of $p$ at lower transferred momenta $Q^{2}$ and where, in general, the non-perturbative terms (41) should saturate and flatten out. Moreover, it was supposed $\|121\|$ that one should use the 
renormalization group improved perturbative expansion and it was shown that this improvement leads to very satisfactory description of experimental data.

Results for jets in $e p$ and $p \bar{p}$ processes also favour the theoretical expectations of the role of coherence effects for emission of soft gluons. Here, analysis is complicated by the internal structure of the colliding objects. Especially interesting are the data [122, 123 on the topology of the $p \bar{p}$-events either with the production of the $W$-meson in combination with the quark or gluon jet or with the production of two hadronic jets 83, 124.

\subsection{Intermittency and fractality}

The self-similar parton cascade leads to special multiparton correlations. Its structure with "jets inside jets inside jets..." caused by the angular ordering has provoked the analogy with turbulence and the ideas of intermittency [125] according to which the increase of fluctuations in ever smaller phase space volume studied (e.g., in smaller rapidity intervals $\delta y$ ) must lead to the increase of factorial moments according to the following power-like behaviour:

$$
F_{q} \propto(\delta y)^{-\phi(q)}
$$

In its turn, this self-similar structure should result in a definite geometric pattern. Namely, this leads to the fractal distribution of particles inside their available phase space [126]. The notion of fractality allows one to quantify the characteristics of the process expressing them in terms of the fractal (or multifractal, Renyi) dimensions. Especially important is the fact that the geometric dimensions are related to the physical characteristics, namely, to the intermittency exponent $\phi(q)$, which shows the slope of the increase of the factorial moments on the doubly logarithmic scale (see Eqn (42)). The slopes (the intermittency exponents) $\phi(q)$ for different ranks $q$ are related to the Renyi dimensions $D_{q}$ in a following way:

$$
\phi(q)=(q-1)\left(D-D_{q}\right)
$$

where $D$ is the topological dimension of the analyzed phase space windows (for example, $D=1$ if the dependence of the factorial moments on the length of the rapidity interval is studied).

To calculate these characteristics, one uses the diagramatic approach in the same way as it was described above when discussing the colour coherence effects. This is necessary now because one has to deal with a small part of the total number of the created partons within the fixed small phase space volume. The fractal behaviour is usually defined by the dependence of the logarithm of the factorial moments in function of the logarithm of the size of the chosen phase space region. The (mono)fractal behaviour would display the linear dependence of logarithms of factorial moments on the logarithmic size of phase space windows. The moments are larger in smaller windows, i.e. the fluctuations increase in smaller bins in a selfsimilar power-like manner if the (mono)fractal distribution is studied (see the review paper [127]).

In QCD, the power dependence of moments on the size of the phase space window appears for a fixed coupling regime [41]. In this case, the monofractal behaviour with a constant Renyi dimension is pronounced:

$$
D_{q}=\frac{q+1}{q} \gamma_{0}
$$

and, correspondingly, the intermittency exponent $\phi(q)=$ const at any fixed rank $q$.

The running coupling strength has a definite scale and, therefore, it leads to some decline from this simple self-similar (monofractal) behaviour. The running property of the coupling strength in QCD flattens off [128, 129, 130] this dependence at smaller bins, i.e. the multifractal behaviour takes over there.

Both the linear (in the doubly logarithmic scale) increase at comparatively large but decreasing 
However, only qualitative agreement with analytical predictions can be claimed here. The higher order calculations are rather complicated and the results of LO with some NLO corrections are yet mostly available. In experiment, the different cuts have been used which hamper the direct comparison. However, those Monte Carlo models where these cuts can be done agree with experiment. The relative role of the partonic and hadronization stages of the cascade in this regime as well as the applicability of the local parton hadron duality hypothesis to the correlation characteristics of the process are still debatable.

The transparent interpretation of the observed effect in terms of the fractal phase space volume has been proposed in the framework of the Lund dipole cascade model [132, 133.

\subsection{The energy behaviour of higher moments of multiplicity distributions}

Differentiating both sides of equations (5), (6) over $u$ and using formulas (26), (27), one can get the equations for the moments of multiplicity distributions of any rank. Their solutions would describe the behaviour of the multiplicity fluctuations. They tell us that the fluctuations of the multiplicity of individual events must be larger for quark jets as compared to gluon jets. Therefore, the moments of their distributions in quark jets are larger than the corresponding moments for gluon jets. This tendency is clearly seen in experimental data [116. The factorial moments increase both with their rank and with energy increasing. In the perturbative expansion one gets the formulas [134], similar to formula (21) for $r$, and the energy increase of the moments is determined by the decrease of $\gamma_{0}$. The corresponding coefficients in front of the terms $\gamma_{0}^{n}$ are calculated in [134]. From the mentioned above behaviour of $H_{q}$-moments one easily guesses that the same is true for the cumulant moments with some difference.

The experimental results for the second rank factorial moments of $41.8 \mathrm{GeV}$ gluon jets $F_{2}^{G}=1.023$ and for $45.6 \mathrm{GeV}$ uds quark jets, $F_{2}^{F}=1.082$ are much smaller than the known long ago (see, e.g., [1]) asymptotic predictions, viz. 1.33 and 1.75 , respectively. The NLO terms improve the description of the data compared to the leading order results. If one accepts the effective value of $\alpha_{S}$ averaged over all the energies of the partons during the jet evolution to be $\alpha_{S} \approx 0.2$, one obtains [134] the NLO values $F_{2}^{G} \approx 1.039$ and $F_{2}^{F} \approx 1.068$ at these energies which are quite close to the experimental results. In this sense the NLO prediction can be said to describe the widths of the gluon and quark jet multiplicity distributions at the $Z^{0}$ energy to within $10 \%$ accuracy.

Unfortunately, the $2 \mathrm{NLO}$ and $3 \mathrm{NLO}$ terms worsen the agreement with data compared to NLO (but not compared to LO) results [134. The same is true for the higher order moments. It raises the general theoretical problem of the convergence of the perturbative expansion in view of the large expansion parameter $q \gamma$ mentioned above. The attempts to account for conservation laws more accurately by the modified evolution equations for high moments [53 have not led to the success yet. It is remarkable that the computer solution of the QCD equations [73] provides a near-perfect description of the higher moments as well. This suggests that the failure of the analytical approach at higher orders is mainly a technical issue related to an inadequate treatment of soft gluons and of energy-momentum conservation. This conclusion is also supported by the success of the Monte Carlo model ARIADNE [135] in describing these characteristics.

The rather accurate experimental results about the multiplicity moments of the separated gluon jets [136] are available now up to the rank 5, and for the quark jets 87] even up to the rank 17, because the accuracy of measuring the multiplicity distributions for the latter is much higher due to the larger statistics of the data.

The higher moments of the multiplicity distributions are determined by the integrals of the correlation functions for partons inside the jet which depend on their angular distributions. The angular correlations have been studied as well [137]. The comparison with experimental data [138, 139] shows that the agreement becomes better with the energy increase even though it is still unsatisfactory at small angles. Some "infrared" stable characteristics like correlations of the energy and multiplicity flows 
have been considered (see, e.g., [140, 141]). They do not require for the determination of the jet axis, as in the case of the angular correlations, where the accuracy of this determination is crucial for the correlation studies at small angles.

In the case of the fixed coupling constant, these equations are exactly solvable [41, 42]. Their solutions as functions of energy possess the scaling property. All moments behave in a power-like manner with energy increasing.

Let us make two short technical comments at the end of this subsection. All analytical expressions should satisfy the requirements of the limiting case of the supersymmetric QCD (the mote detailed discussion of this problem and the corresponding formulas for the coefficients $a_{i}$ and $r_{i}$ from the Table 1 see in Ref. [72]). The factorial moments are always positive according to their definition. These two requirements are the necessary but not sufficient conditions for the correctness of the calculations. However, the moments calculated in Ref. [78] do not satisfy the second condition. At the same time, the coefficient $r_{2}$ obtained in Ref. [77] satisfies the requirements of the supersymmetric QCD (as well as that from Ref. [72 which differs numerically) but the method of the renormalization group used in Ref. [77] does not take into account correctly the energy-momentum conservation laws and gives rise to the numerically smaller value of this coefficient.

\subsection{Subjet multiplicities}

A single quark-antiquark pair is initially created in $e^{+} e^{-}$-annihilation. With very low angular resolution (large angle averaging) one observes two jets. A three-jet structure can be observed if a gluon with large transverse momentum is emitted by the quark or antiquark. However such a process is suppressed by an additional factor $\alpha_{S}$, which is small for large transferred momenta. Its probability can be calculated perturbatively. At relatively low transferred momenta, the jet evolves to the angular ordered subjets ("jets inside jets inside jets..."). Different algorithms have been proposed to resolve subjets. By increasing the resolution, more and more subjets are observed. For very high resolution, the final hadrons are resolved. The resolution criteria are chosen to provide the infrared safe results (see, e.g., the papers [64, 142, 143, 144, 145, 146]).

In particular, one can predict the asymptotic ratio of subjet multiplicities in 3- and 2-jet events if one neglects the soft gluon coherence:

$$
\frac{n_{3}^{s j}}{n_{2}^{s j}}=\frac{2 C_{F}+C_{A}}{2 C_{F}}=\frac{17}{8} .
$$

Actually, the coherence reduces this value to be below 1.5 in experiment for all acceptable resolution parameters. The theoretical predictions [70] agree quantitatively with experimental findings [147, 148] at the lowest resolution, where this ratio is equal to $3 / 2$, and only qualitatively at higher resolutions. The computer calculations [73, 74] agree quite well with experiment for different resolution parameters that implies the importance of the precise account for the energy-momentum conservation.

Subjet multiplicities have also been studied for the separated quark and gluon jets. The analytical results [149] are seen (Fig. 12 from [150]) to represent the data fairly well for large values of the subjet resolution scale $y_{0}$.

\subsection{Jet universality}

According to QCD, jets produced in processes initiated by different colliding particles must be universal and depend only on their own parent (gluon, light or heavy quark). This prediction has been confirmed by many experiments. For example, this is clearly seen if the multiplicities in the fragmentation region in the Breit system of $e p$-collisions are compared with the corresponding multiplicities in $e^{+} e^{-}$-processes at high energies. It has been found [151] that they coincide as is required by the universality condi- 
demonstrated in Fig. 8. Therefore we will not discuss it at some length but just stress once again the importance of such a prediction and its non-trivial origin. At the same time, let us mention that the universality of the properties of the jets produced does not imply the universality of the mechanisms of their production in various processes.

\section{Conclusions and outlook}

A list of successful analytical QCD predictions can be made longer. As was demonstrated above, quantum chromodynamics has already predicted spectacular qualitative features of rather soft processes. Quantitatively, analytical results show that the higher order (NLO) terms always tend to improve the agreement with experiment compared to the asymptotic (LO) predictions. The accuracy achieved is often better than $20 \%$ or even $10 \%$ that is surprising by itself considering the rather large values of the expansion parameter of the perturbative approach. Moreover, some characteristics are very sensitive to ever higher order terms and should be carefully studied. The astonishing success of the computer solutions and Monte Carlo schemes demonstrates the importance of using the correct borderline between the perturbative and non-perturbative regions, which is approximately accounted in the analytical perturbative approach by the cut-off parameter $Q_{0}$ and by the limits of integration over parton splitting variables. One can expect that the purely perturbative description becomes dual to the sum over all possible hadronic excitations. Nevertheless, the correspondence between the parton stage of the cascade evolution and the hadronization is not always defined at the quantitative level and requires further studies and the development of the common point of view. In particular, this problem is approached in a different way not only in analytical results if they are compared with the Monte Carlo models' conclusions but also in different Monte Carlo schemes.

A new era of multiparticle production studies opens with the advent of new accelerators RHIC, LHC, TESLA, NLC, JLC, CLIC. We come closer to the asymptotic region [3] even though the approach to the asymptotic laws will be, probably, extremely slow because all the predicted energy dependences of the physically measurable quantities are slow as well. Nevertheless, some predictions differ for various analytical approaches and Monte Carlo schemes at these energies and will be confronted to experimental data. It will allow to distinguish between them. The qualitative QCD predictions indicate the tendencies towards the asymptotic region where the perturbative estimates become more precise.

The mean multiplicities will increase drastically. Now, in Au-Au collisions at the center of mass energies of $130 \mathrm{GeV}$ per nucleon at RHIC the mean charged multiplicity exceeds 4000. It implies that the event-by-event analysis of various patterns formed by particles in the available phase space becomes meaningful. Such an analysis would allow one to classify in more detail the multiparticle production processes than, say, it is done in hadronic interactions where simple separation of the diffractive and non-diffractive processes is only considered. The study of the topology of individual events must provide us with a much richer information on the dynamics of the process compared with the results of the measurement of characteristics averaged over the whole event sample. Its results can be compared to the exclusive probabilistic Monte Carlo schemes. Searches for the supersymmetric partners of well known particles, Higgses, new states of matter, new collective and interference effects, physics of jets and mini-jets will, surely, be among the most important directions of further investigations.

The event-by-event approach would allow one to analyze the small colour-suppressed effects, which show the difference between the perturbative QCD and Monte Carlo calculations for the topology of

\footnotetext{
${ }^{13}$ Let allow me to make here some short historical and lyrical digression. About 45 years ago, the tuitor of my diploma work I.Ya. Pomeranchuk told me that with the advent of the $10 \mathrm{GeV}$ Dubna accelerator we enter the asymptotic region because $10 \gg 1$ (By 1 he meant the nucleon mass.). Now, we understand that this hope was too naive. Probably, our today's feelings are not more satisfactory even though they are supported by the modern QCD predictions. However, possible effects of the high parton density and of the non-linear interactions, which nowadays escape the detailed treatment, could change the situation at high energies.
} 
the individual events, properties of minijets or clusters (with the attraction-repulsion transition), other collective effects like the elliptic flow (and even the higher Fourier expansion terms of the azimuthal distribution in an individual event), the possible azimuthal asymmetry of the opening angle for individual jets, the ring-like events (the probable signature of the confinement and/or of the "Cherenkov gluons"), further analysis of the difference in accompanying gluon emission by heavy and light quarks (in particular, the angular distribution difference) etc.

Of the principal importance is the study of the multiparticle correlations. The use of traditional formulas for the correlation functions is hampered by the large number of the independent variables. One can overcome this difficulty in spite of the seemingly complicated structure of these functions. The event-by-event analysis of experimental exclusive data can become available, quantified locally and provide statistically significant results if one uses wavelets (sometimes called the "mathematical microscope") for the pattern recognition in individual events 152. With the help of wavelets one can separate correlations at the different resolution levels (from the short-range to long-range correlations) locally and in a compact form. In principle, the distributions of the wavelet coefficients can replace the complicated expressions for the multiparticle correlators.

At the same time, the intriguing data obtained in the cosmic ray studies (for the recent review see Ref. [153) tell us that one can await for new phenomena in the fragmentation region of hadronic (and nuclear) collisions which, unfortunately, has not been devoted sufficient attention in accelerator experiments because most detectors are suited for the central range of rapidities. In particular, it would be important to learn the ratio between the baryon, meson and photon contents of the multiparticle production events.

To confront QCD predictions with new experimental findings at ever higher energies will be crucial for the search for possible new states of matter. This is also important for the separation of any new physical signals from the conventional QCD background.

\section{Acknowledgements}

I am deeply indebted to all colleagues with whom I worked on the problems reviewed in this paper, and to the authors of those numerous papers which often stimulated this research. This work was supported by the RFBR grant 00-02-16101.

\section{References}

[1] Andreev I V Chromodynamica i zhestkie processy pri vysokih energiyah (Moscow, Nauka, 1981) (in Russian)

[2] Ioffe B L, Lipatov L N and Khoze V A Glubokoneuprugie processy (Moscow, Energoatomizdat, 1983) (in Russian)

[3] Yndurain F J Quantum chromodynamics (N.-Y.-Berlin-Heidelberg-Tokyo, Springer Verlag, 1983).

[4] Voloshin M B and Ter-Martirosyan K A Teoriya kalibrovochnyh vzaimodeistvii elementarnyh chastits (Moscow, Energoatomizdat, 1984) (in Russian)

[5] Dokshitzer Yu L, Khoze V A, Mueller A H and Troyan S I Basics of perturbative QCD ed. J. Tran Thanh Van (Gif-sur-Yvette, Editions Frontieres, 1991).

[6] Dremin I M UFN 164785 (1994); Phys.-Uspekhi 37715 (1994)

[7] Khoze V A and Ochs W Int. J. Mod. Phys. A 122949 (1997) 
[9] Khoze V A, Ochs W and Wosiek J in "Handbook of QCD" (Ioffe Festschrift) (WSPC, Singapore) (to be published); hep-ph/0009298

[10] Stirling W J J. Phys. G 26471 (2000)

[11] Gribov L V, Levin E M and Ryskin M G Phys. Rep. 1001 (1983)

[12] Levin E M and Ryskin M G Phys. Rep. 189267 (1990)

[13] Lipatov L N Phys. Rep. 286131 (1997)

[14] Frankfurt L L and Strikman M I Phys. Rep. 160235 (1988)

[15] Wang X-N Phys. Rep. 280287 (1997)

[16] Schmidt C R Proc. RADCOR 2000, Santa Cruz, CA, USA, 11-16 Sept 2000; hep-ph/0106181

[17] Andersson B, Gustafson G, Ingelman G and Sjöstrand T Phys. Rep. 9731 (1983)

[18] Marchesini G and Webber B Nucl. Phys. B 2381 (1984); B 310461 (1988)

[19] Marchesini G, Webber B, Abbiendi G et al Comp. Phys. Comm. 43465 (1992)

[20] Azimov Ya I, Dokshitzer Yu L, Khoze V A and Troyan S I Z. Phys. C 2765 (1985); C 31213 (1986)

[21] Amati D and Veneziano G Phys. Lett. B 8387 (1979)

[22] Bassetto A, Ciafaloni M and Marchesini G Phys. Lett. B 83207 (1979)

[23] Marchesini G, Trentadue L and Veneziano G Nucl. Phys. B 181335 (1981)

[24] Konishi K, Ukawa A and Veneziano G Nucl. Phys. B 15745 (1979)

[25] Mueller A H Nucl. Phys. B 21385 (1983); Erratum B 241141 (1984)

[26] Gribov V N and Lipatov L N YaF 151218 (1972); Sov. J. Nucl. Phys. 15781 (1972)

[27] Altarelli G and Parisi G Nucl. Phys. B 126298 (1977)

[28] Dokshitzer Yu L ZhETF 731216 (1977); Sov. Phys. JETP 73641 (1977)

[29] Lipatov L N YaF 23642 (1976); Sov. J. Nucl. Phys. 23338 (1976)

[30] Kuraev E A, Lipatov L N and Fadin V S ZhETf 72377 (1977); Sov. Phys. JETP 72199 (1977)

[31] Balitsky Ya Ya and Lipatov L N YaF 281597 (1978); Sov. J. Nucl. Phys. 28822 (1978)

[32] Ciafaloni M Nucl. Phys. B 29649 (1988);

Catani S, Fiorani F and Marchesini G Phys. Lett. B 234339 (1990); Nucl. Phys. B 33618 (1990)

[33] Gustafson G Proc. 30 Int. Symp. on Multiparticle Dynamics, Tihany, Hungary, 9-15 Oct 2000, Eds T Csorgo, S Hegyi, W Kittel, World Scientific, Singapore, 2001, p 42;

Proc. 31 Int. Symp. on Multiparticle Dynamics, Datong, China, 1-7 Sept 2001 (to be published)

[34] Lipatov L N Nucl Phys Proc Suppl 99A 175 (2001)

[35] De Vega and Lipatov L N hep-ph/0107225 
[36] McLerran L and Venugopalan R Phys. Rev. D 493352 (1994)

[37] Jalilian-Marian J, Kovner A, Leonidov A and Weigert H Nucl. Phys. B 504415 (1997)

[38] Leonidov A Proc. Conf. "Quantum Field Theory and Strings", Moscow, June 2000 (to be published)

[39] Jalilian-Marian J, Kovner A and Weigert H Phys. Rev. D 59014015 (1999)

[40] Dremin I M Phys. Lett. B 313209 (1993)

[41] Dremin I M and Hwa R C Phys. Rev. D 495805 (1994)

[42] Dremin I M and Hwa R C Phys. Lett. B 324477 (1994)

[43] Shirkov D V TMF 127409 (2001)

[44] Marchesini G Nucl. Phys. Proc. Suppl. 7185 (1999)

[45] Dokshitzer Yu L, Marchesini G and Webber B R JHEP 9907:012 (1999)

[46] Banfi A, Dokshitzer Yu L, Marchesini G and Zanderighi G JHEP 0007:002 (2000); Phys. Lett. B508 269 (2001); JHEP 0103:007 (2001)

[47] Dremin I M Pis'ma v ZhETF 31215 (1980); JETP Lett. 31185 (1980)

[48] Dremin I M and Leonidov A V YaF 35288 (1982); Sov. J. Nucl. Phys. 35247 (1982)

[49] Leonidov A V and Ostrovsky D M YaF 60185 (1997); Phys. Atom. Nucl. 60119 (1997)

[50] Ellis J and Geiger K Phys. Rev. D 521500 (1995)

[51] Ellis J and Geiger K Nucl. Phys. A 590 609c (1995)

[52] Eden P Proc. XXXIV Moriond conf. "QCD and strong interactions" March 1999, ed. J. Tran Thanh Van (Editions Frontieres, Gif-sur-Yvette, 1999)

[53] Dremin I M and Eden P Proc. 31 Int. Symp. on Multiparticle Dynamics (Datong, China, Sept 2001) (to be published)

[54] Yang C N and Lee T D Phys. Rev. 87404 (1952)

[55] Lee T D and Yang C N Phys. Rev. 87410 (1952)

[56] Particle Data Group, Barnett R M et al Phys. Rev. D 541 (1996)

[57] CLEO Collaboration, Alam M S et al Phys. Rev. D 464822 (1992); Phys. Rev. D 5617 (1997)

[58] Dokshitzer Yu L, Troyan S I and Khoze V A YaF 471010 (1988); Sov. J. Nucl. Phys. 47881 (1988)

[59] Gary J W Phys. Rev. D 494503 (1994)

[60] OPAL Collaboration, Alexander G et al Phys. Lett. B 388659 (1996)

[61] Eden P and Gustafson G JHEP 9809015 (1998)

[62] Eden P, Gustafson G and Khoze V A Eur. Phys. J. C 11345 (1999) 
[63] Gary J W in Proc. 31 Int. Symp. on Multiparticle Dynamics 1-7 Sept. 2001, Datong, China, Eds. Liu L., Wu Y., World Scientific, Singapore, 2002 (to be published);

OPAL Collaboration Physics Note PN488, 2001

[64] Brown N and Stirling W J Phys. Lett. B 252657 (1990); Z. Phys. C 53629 (1992)

[65] Mueller A H Phys. Lett. B 104161 (1981)

[66] Dokshitzer Yu L, Fadin V S and Khoze V A Z. Phys. C 15335 (1982); C 1883 (1983)

[67] Bassetto A, Ciafaloni M, Marchesini G and Mueller A H Nucl. Phys. B 207189 (1982)

[68] Webber B R Phys. Lett. B 143501 (1984)

[69] Dokshitzer Yu L, Khoze V A and Troyan S I Int. J. Mod. Phys. A 71875 (1992)

[70] Catani S, Dokshitzer Yu L, Fiorani F and Webber B R Nucl. Phys. B 377445 (1992)

[71] Dremin I M and Gary J W Phys. Lett. B 459341 (1999)

[72] Capella A, Dremin I M, Gary J W, Nechitailo V A and Tran Thanh Van J Phys. Rev. D 61074009 (2000)

[73] Lupia S Phys. Lett. B 439150 (1998); Proc. XXXIII Moriond conf. "QCD and strong interactions" March 1998, ed. J. Tran Thanh Van (Editions Frontieres, Gif-sur-Yvette, 1998) p. 363

[74] Lupia S and Ochs W Phys. Lett. B 418214 (1998); Nucl. Phys. (Proc. Suppl.) B 6474 (1998)

[75] Brodsky S J and Gunion J F Phys. Rev. Lett. 37402 (1976)

[76] Mueller A H Nucl. Phys. B 241141 (1984)

[77] Gaffney J B and Mueller A H Nucl. Phys. B 250109 (1985)

[78] Malaza E D and Webber B R Nucl. Phys. B 267702 (1986)

[79] Dremin I M and Nechitailo V A Mod. Phys. Lett. A 91471 (1994); JETP Lett. 58881 (1993)

[80] Dremin I M Pis'ma v ZhETF 68635 (1998); JETP Lett. 68559 (1998)

[81] DELPHI Collaboration, Abreu P et al Phys. Lett. B 449383 (1999)

[82] OPAL Collaboration, Abbiendi G et al CERN-EP-2000-070

[83] Khoze V A, Lupia S and Ochs W Eur. Phys. J. C 577 (1998)

[84] Khoze V A, Lupia S and Ochs W Phys. Lett. B 394179 (1997)

[85] OPAL Collaboration, Abbiendi G Eur. Phys. J. C 11217 (1999)

[86] Dremin I M, Arena V, Boca G et al Phys. Lett. B 336119 (1994)

[87] SLD Collaboration, Abe K et al Phys. Lett. B 371149 (1996)

[88] Dremin I M, Nechitailo V A, Biyajima M and Suzuki N Phys. Lett. B 403149 (1997)

[89] Dremin I M Phys. Lett. B 34195 (1994) 
[91] Brooks T C, Kowalski K L and Taylor C C Phys. Rev. D 565857 (1997)

[92] Azimov Ya I, Dokshitzer Yu L and Khoze V A Pis'ma v ZhETF 35390 (1982); JETP Lett. 35482 (1982)

[93] Fong C P and Webber B R Phys. Lett. B 229289 (1989); B 241255 (1990); Nucl. Phys. B 355 $54(1991)$

[94] DELPHI Collaboration, Abreu P et al Phys. Lett. B 459397 (1999)

[95] CDF Collaboration, Safonov A N Nucl. Phys. (Proc. Suppl.) B 8655 (2000)

[96] Lupia S and Ochs W Phys. Lett. B 365339 (1996)

[97] Lupia S and Ochs W Eur. Phys. J. C 2307 (1998)

[98] Dremin I M, Nazirov M T and Saakian V A YaF 421010 (1985); Sov. J. Nucl. Phys. 42845 (1985)

[99] Dokshitzer Yu L, Khoze V A and Troyan S I J. Phys. G 17 1481, 1602 (1991)

[100] Schumm B A, Dokshitzer Yu L, Khoze V A and Koetke D S Phys. Rev. Lett. 693025 (1992)

[101] Petrov V A and Kisselev A V Z. Phys. C 66453 (1995)

[102] Azimov Ya I, Dokshitzer Yu L and Khoze V A 361510 (1982); Sov. J. Nucl. Phys. 36878 (1982)

[103] Kisselev A V, Petrov V A and Yuschenko O P Z. Phys. C 41521 (1988)

[104] DELPHI Collaboration, Abreu P et al Phys. Lett. B 479118 (2000); Erratum - ibid B 492398 (2000)

[105] DELPHI Collaboration, Abreu P et al Z. Phys. C 57181 (1993);

ALEPH Collaboration, Busculic D et al Phys. Lett. B 357699 (1995);

OPAL Collaboration, Alexander G et al Phys. Lett. B 36493 (1995);

OPAL Collaboration, Akers R et al Z. Phys. C 6727 (1995);

SLD Collaboration, Abe K et al Phys. Rev. Lett. 844300 (2000)

[106] Dremin I M and Yakovlev V I Proc. 17 Int. Symp. on Multiparticle Dynamics Ed. M. Markytian, Austria, 1986 (World Scientific, Singapore, 1987) p 849

[107] OPAL Collaboration, Ackerstaff K et al Eur. Phys. J. C 7369 (1999)

[108] DELPHI Collaboration, Nomokonov V Proc. Int. Europhysics Conf. HEP99, Tampere, Finland, July 1999; hep-ex/9910059

[109] Dremin I M Pis'ma v ZhETF 30154 (1979), JETP Lett. 30140 (1979); YaF 331357 (1981), Sov. J. Nucl. Phys. 33726 (1981)

[110] Dremin I M, Ivanov O V, Kalinin S A et al Phys. Lett. B 49997 (2001)

[111] Chudakov A E Izv. AN SSSR, ser fiz 19650 (1955)

[112] DELPHI Collaboration, Abreu P et al Z. Phys. C 5663 (1992)

[113] Gary J W Phys. Rev. D 61114007 (2000)

[114] Andersson, Gustafson G and Sjöstrand T Phys. Lett. B 94211 (1980) 
[115] Azimov Ya I, Dokshitzer Yu L, Khoze V A and Troyan S I Phys. Lett. B 165147 (1985); 43149 (1986); Sov. J. Nucl. Phys. 4395 (1986)

[116] DELPHI Collaboration, Abreu P et al Z. Phys. C 70179 (1996)

[117] Dokshitzer Yu L, Khoze V A and Troyan S I YaF 461220 (1987); Sov. J. Nucl. Phys. 46712 (1987)

[118] DELPHI Collaboration, CERN-OPEN-2000-134

[119] Beneke M Phys Rep 3171 (1999)

[120] Dokshitzer Yu L Talk at IPPP Workshop on Multiparticle Production in QCD Jets, Durham, England, Dec. 2001

[121] Hamacher K Talk at IPPP Workshop on Multiparticle Production in QCD Jets, Durham, England, Dec. 2001

[122] Khoze V A and Stirling W J Z. Phys. C 7659 (1997)

[123] D0 Collaboration, Abbott B et al Phys. Lett. B 414419 (1997)

[124] Butterworth J M, Khoze V A and Ochs W J. Phys. G 251457 (1999)

[125] Bialas A and Peschanski R Nucl. Phys. B 273703 (1986)

[126] Dremin I M Pis'ma v ZhETF 45505 (1987); JETP Lett. 45643 (1987)

[127] DeWolf E A, Dremin I M and Kittel W Phys. Rep. 2701 (1996)

[128] Dokshitzer Yu L and Dremin I M Nucl. Phys. B 402139 (1993)

[129] Ochs W and Wosiek J Phys. Lett. B 289159 (1992); B 304144 (1993)

[130] Brax Ph, Meunier J L and Peschanski R Z. Phys. C 62649 (1994)

[131] OPAL Collaboration, Abbiendi G et al Eur. Phys. J. C 11239 (1999)

[132] Andersson B, Dahlquist P and Gustafson G Phys. Lett. B 214604 (1988)

[133] Dahlquist P, Andersson B and Gustafson G Nucl. Phys. B 23876 (1989)

[134] Dremin I M, Lam C S and Nechitailo V A Phys. Rev. D 61074020 (2000)

[135] Lupia S, Ochs W and Wosiek J Nucl. Phys. B 540405 (1999)

[136] OPAL Collaboration, Ackerstaff K et al Eur. Phys. J. C 1479 (1998)

[137] Ochs W and Wosiek J Phys. Lett. B 304144 (1993)

[138] DELPHI Collaboration, Abreu P et al Phys. Lett. B 440203 (1998)

[139] ZEUS Collaboration, Breitweg et al Eur. Phys. J. C 1253 (2000)

[140] Dokshitzer Yu L, Khoze V A, Marchesini G and Webber B R Phys. Lett. B 245243 (1990)

[141] Ochs W and Wosiek J Z. Phys. C 72263 (1996) 
[143] Stirling W J J. Phys. G 171567 (1991)

[144] Sjöstrand T Comp. Phys. Comm. 28229 (1983)

[145] JADE Collaboration, Bartel W et al Z. Phys. C 3323 (1986); Bethke S et al Phys. Lett. B 213 235 (1988)

[146] Dokshitzer Yu L, Leder G D, Moretti S and Webber B R JHEP 97081 (1997)

[147] L3 Collaboration, Adriani O et al Phys. Rep. 2361 (1993)

[148] OPAL Collaboration, Akers R et al Z. Phys. C 63363 (1994)

[149] Seymour M H Phys. Lett. B 378279 (1996)

[150] ALEPH Collaboration, Busculic D et al Phys. Lett. B 346389 (1995)

[151] H1 Collaboration, Adloff C et al Nucl. Phys. B 5043 (1997)

[152] Dremin I M, Ivanov O V and Nechitailo V A UFN 171465 (2001); Phys.-Uspekhi 44 (5) (2001).

[153] Gladysz-Dziadus E Elem. Part. Atom. Nucl. (2002) (to be published)

\section{Figure captions}

Fig. 1. The energy dependence of average multiplicity of charged particles in $e^{+} e^{-}$-annihilation. The results of different fits according to formulas of perturbative QCD and of the Monte Carlo models are shown ( the solid and dotted lines are the fits of formula (23) with one and two adjusted parameters, the dashed line is given by the HERWIG Monte Carlo model; the vertically shaded area indicates the gluon jet data multiplied by the theoretical value of the ratio $r(21)$ ).

Fig. 2. The experimentally measured ratio $r$ of multiplicities in gluon and quark jets as a function of energy in comparison with the predictions of analytical QCD and of the Monte Carlo model HERWIG (different QCD approximations, described in this paper, as well as $3 \mathrm{NLO}(\epsilon)$ with integration limits $e^{-y}$ and $1-e^{-y}$ in Eqns (5), (6) are indicated at the corresponding lines).

Fig. 3. The ratio of the slopes of the energy dependences of mean multiplicities in gluon and quark jets according to experimental data and some theoretical calculations.

Fig. 4. The ratio of the curvatures of the energy dependences of mean multiplicities in gluon and quark jets according to experimental data and some theoretical calculations.

Fig. 5. The measured ratio $H_{q}$ of the cumulant and factorial moments oscillates as a function of the rank $q$ according to experimental data on multiplicity distributions of charged particles in $e^{+} e^{-}$annihilation at the $Z^{0}$ energy (the inset in the upper right corner shows the data for the moments of the ranks 2,3 and 4 ).

Fig. 6. The inclusive rapidity distribution of secondary particles in $e^{+} e^{-}$-annihilation has the shape of the hump-backed plateau (it is shown in the center of mass system).

Fig. 7. The peak in the variable $\xi$ is fitted at different energies by the distorted Gaussian with the moments predicted by the NLO-approximation of perturbative QCD (the fitted lines are drawn at $\Lambda=210$ ) according to 93]). 
Fig. 8. The peak position of the experimental inclusive distribution as a function of the mass of two jets is compared with NLO predictions (the central line is fitted by the CDF Collaboration data only). The lowest order (LO) predictions are shown by the lower straight line. The expected behaviour without the colour coherence effect gives rise to the upper line.

Fig. 9. The energy dependence of the difference between the average multiplicities of charged particles in events initiated by $b$ and $(u, d, s)$-quarks. Experimental dots are compared with QCD predictions (the horizontal stripe) and with the results of the naive rescaling model (the decreasing stripe).

Fig. 10. The charged hadronic flows in 3-jet events (the histogram) in comparison with analytical QCD predictions (the solid line) as functions of the azimuthal angle.

Fig. 11. The normalized factorial moments of various ranks as functions of their scale (the size of the phase space window diminishes to the right on the abscissa axis). Different analytical approximations are compared with experimental data. LO-approximation (DLLA): (a) - [128], (b) - [130], (c) - [129]; NLO-approximation (MLLA) - 128. The qualitative but not quantitaive agreement is seen.

Fig. 12. The subjet multiplicities in the separated gluon (a) and quark (b) jets as functions of the resolution parameter $y_{0}$ are compared with analytical QCD results and with predictions of the Monte Carlo model JETSET for hadrons (HL) and partons (PL). 